\title{
CLASSIFICAÇÃO CLIMÁTICA DE KÖPPEN E DE THORNTHWAITE PARA MINAS GERAIS: CENÁRIO ATUAL E PROJEÇÕES FUTURAS
}

\author{
MARTINS, Fabrina Bolzan - fabrinabm@gmail.com \\ Universidade Federal de Itajubá / UNIFEI \\ GONZAGA, Gabriela - gabi-gonzaga@live.com \\ Universidade Federal de Itajubá / UNIFEI
}

\author{
SANTOS, Diego Felipe dos - dfsantos17@hotmail.com \\ Universidade Federal de Itajubá / UNIFEI
REBOITA, Michelle Simões - mireboita@gmail.com Universidade Federal de Itajubá / UNIFEI

\begin{abstract}
RESUMO: O estado de Minas Gerais (MG) possui representatividade nacional em termos de extensão territorial, contingente populacional e contribuição na economia do país. Para o final deste século, projeta-se um clima mais quente que pode afetar diretamente as atividades da população e a economia local. Portanto, o objetivo do presente estudo foi determinar os Sistemas de Classificação Climática (SCC) de Köppen e de Thornthwaite para MG no clima presente (1981 a 2010) e futuro (2011 a 2100). Para tanto, foram utilizados dados de temperatura do ar e precipitação observados e projetados por modelos climáticos globais. Pelo SCC de Köppen, há cinco tipos climáticos em MG: dois temperados quentes (Cwb, Cwa), um tropical (Aw) e dois semiáridos (BSh e BWh); já pelo SCC de Thornthwaite há vinte e cinco tipos climáticos com predominância do tipo megatérmico e mesotérmico superúmido e úmido concentrados nas regiões Central, da Mata, Sul, Paranaíba e Centro-Oeste, e megatérmico e mesotérmico subúmido ou semiárido nas demais regiões de MG. Independente do SCC, MG apresenta duas características climáticas bem definidas nas projeções futuras: nas regiões centrais do estado os tipos climáticos atuais se mantêm enquanto que nos extremos norte e sul do estado, devido à tendência de aumento da temperatura e redução da umidade relativa do ar, há alteração para tipos climáticos mais quentes e secos.
\end{abstract}

PALAVRAS-CHAVE: sistemas de classificação climática, mudanças climáticas, modelos climáticos globais

\section{CLIMATE CLASSIFICATION OF KÖPPEN AND THORNTHWAITE FOR MINAS GERAIS: CURRENT CLIMATE AND CLIMATE CHANGES PROJECTIONS}

ABSTRACT: Minas Gerais (MG) State presents great national representativeness in terms of territorial extension, population contingent and contribution in the economy of the country. Towards the end of this century, a warmer climate is projected that can directly affect the activities of the population and the local economy. Therefore, the objective of this study was to determine two Climate Classification Systems (CCS) - Köppen and Thornthwaite - for MG in the present climate (1981 to 2010) and in the future climate (2011 and 2100). For this reason, we used air temperature and precipitation data observed and projected by global climate models. According to the CCS of Köppen there are five climatic types in MG: two warm temperate (Cwb, Cwa), one tropical (Aw) and two semi-arid ones (BSh and BWh); to CCS of Thornthwaite there are twenty-five climatic types with predominance of the megathermic and mesothermic type superhumid and humid concentrated in the Central, Mata, Sul, Paranaíba and Center-West regions, and megathermic and mesothermic subhumid or semi-arid in the other regions of MG. Regardless of the CCS, MG presents two well-defined climate characteristics in future projections: in the central regions of the state, the current climate types are maintained while in the north and south extremes of the state, due to the tendency of temperature increase and reduction of humidity, there is a change to hotter and drier climate types. 
KEYWORDS: climate classification system, climate change, global climate models

CLASIFICACIÓN CLIMÁTICA DE KÖPPEN Y DE THORNTHWAITE PARA MINAS GERAIS: ESCENARIO ACTUAL Y PROYECCIONES CLIMATICAS

RESUMEN: El estado de Minas Gerais (MG) posee representatividad nacional en extensión territorial, contingente poblacional y contribución en la economía del país. A finales del siglo XXI, se proyecta un clima más cálido que podrán afectar las actividades de la población y la economía. El objetivo del estudio fue determinar los Sistemas de Classificación Climática (SCC) de Köppen y de Thornthwaite para MG en el clima actual (1981 a 2010) y futuro (2011 a 2100). Se utilizaron dados de temperatura del aire y precipitación observados y proyectados por modelos climáticos globales. Por el SCC de Köppen, hay cinco tipos climáticos en MG: dos templados (Cwb, Cwa), un tropical (Aw) e dos semiáridos (BSh e BWh); ya por el SCC de Thornthwaite hay 25 tipos climáticos con predominio del tipo megatérmico y mesotérmico superúmedo y húmedo concentrados en las regiones Central, Mata, Sul, Paranaíba y Centro-Oeste, y megatérmico y mesotérmico subúmedo o semiárido en las demás regiones de MG. En los dos SCC, MG presenta dos características climáticas bien definidas en las proyeciones futuras: en las regiones centrales los tipos climáticos actuales se mantienen mientras en los extremos norte y sur, debido a la tendencia de aumento de temperatura e reducción da precipitacíon, hay câmbios para tipos climáticos más cálidos y secos.

PALABRAS CLAVE: sistemas de classificación climática, câmbios climáticos, modelos climáticos globais

\section{INTRODUÇÃO}

Os Sistemas de Classificação Climática (SCC) são utilizados a fim de sintetizar as características climáticas de um dado local. Esses sistemas ajudam a identificar espacialmente as áreas com características climáticas similares. Os SCC são bastante empregados em estudos associados à agricultura objetivando compor informações em estudos de zoneamento agroclimático (ALVARES et al., 2014), zoneamento de espécies florestais (ARAÚJO et al., 2012; GONÇALVES et al., 2013), risco de doença em espécies florestais (SILVA et al., 2013), alterações nos biomas (FERNANDEZ et al., 2017) entre outros.

Dentre os SCC mais utilizados têm-se o de Köppen (1936) e o de Thornthwaite (1948). O SCC de Köppen utiliza dados médios mensais de temperatura do ar e precipitação na definição dos limites climáticos (CUNHA; MARTINS, 2009; REBOITA et al., 2015). Esse SCC é conhecido pela simplicidade, aplicabilidade e facilidade de interpretação dos resultados (VIANELLO; ALVES, 2012). No Brasil, a utilização do SCC de Köppen é comum pelo Instituto Brasileiro de Geografia e Estatística (IBGE) e por outros órgãos estaduais. Já o SCC de Thornthwaite é baseado em médias climatológicas mensais do extrato do balanço hídrico climatológico (BHC), temperatura do ar e precipitação (ROLIM et al., 2007; NÓBREGA, 2010). A vantagem desse SCC comparado ao SCC de Köppen é a maior sensibilidade na definição dos limites climáticos, pois detecta pequenas variações espaciais com maior eficiência (CUNHA; MARTINS, 2009). Por outro lado, uma desvantagem do SCC de Thornthwaite é sua maior complexidade, devido à necessidade de calcular o BHC (FABRES, 2009).

O estado de Minas Gerais (MG) é o segundo mais populoso do país (20.997.560 habitantes) e o quarto maior em extensão territorial (586.521,235 $\mathrm{km}^{2}$ de área) (IBGE, 2016); possui cerca de $1 / 3$ do produto interno bruto (PIB) dependente do setor agrícola. Por esses motivos, mudanças no clima alterarão a qualidade de vida da população e a economia mineira. Nesse contexto, esforços devem ser realizados no sentido de avaliar e comparar os SCC para MG 
considerando o clima presente, bem como as projeções futuras. Salienta-se a escassez de estudos dessa natureza para MG, pois os de Sá Junior (2009) e Reboita et al. (2015) consideram apenas o clima presente. O único estudo (encontrado até o momento) que mostra projeções climáticas é o de Fernandez et al. (2017) que avaliaram as projeções do SCC de Köppen-Trewartha sobre toda a América do Sul, através de um modelo climático regional. Diante desse contexto, o objetivo do estudo é computar e comparar os SCC de Köppen e de Thornthwaite, considerando o clima presente e futuro, no estado de MG.

\section{MATERIAL E MÉTODOS}

\section{1 ÁREA DE ESTUDO E DADOS}

Inicialmente foram determinados os SCC para o clima presente (19812010) a partir de dados diários de precipitação acumulada ( $\mathrm{mm}$ ) e temperatura máxima e mínima do ar $\left({ }^{\circ} \mathrm{C}\right)$ de 49 estações meteorológicas convencionais (Figura 1), pertencentes ao Instituto Nacional de Meteorologia. Com esses dados, calcularam-se as médias climatológicas mensais das variáveis atmosféricas no referido período. Nesse cálculo foram utilizados apenas os meses com mais de $85 \%$ de dados válidos, isto é, meses com menos de 5 dias faltantes aproximadamente (WMO, 1989). Todas as 49 estações meteorológicas apresentaram no mínimo 10 anos de dados válidos em todos os meses do ano.

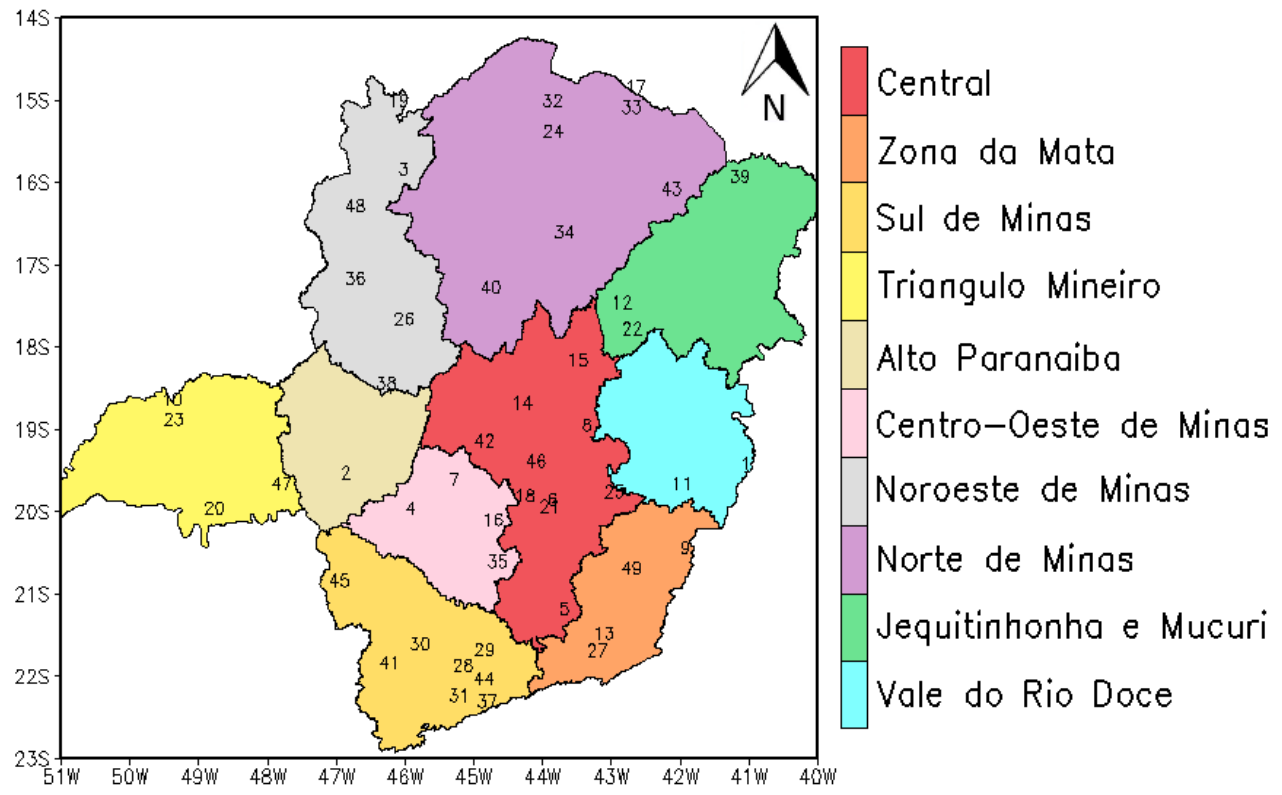

Figura 1 - Localização das estações meteorológicas convencionais utilizadas no estudo em cada região. Adaptado de Garcia et al. (2018).

Para o cálculo dos SCC e das estatísticas, optou-se por dividir o estado em suas regiões de planejamento administrativo (Tabela 1 e Figura 1; IBGE, 2016): Central (Região I), Zona da Mata (Região II), Sul de Minas (Região III), Triângulo Mineiro (Região IV), Alto Paranaíba (Região V), Centro-Oeste de Minas (Região VI), Noroeste de Minas (Região VII), Norte de Minas (Região VIII), Jequitinhonha e Mucuri (Região IX) e Vale do Rio Doce (Região X). As regiões $V$ e $\mathrm{X}$ apresentavam duas ou menos estações meteorológicas, sendo impossível 
realizar os testes estatísticos. Portanto, essas regiões foram agrupadas nas regiões mais próximas, sendo a região $\mathrm{V}$ agrupada com a região $\mathrm{IV}$ e a região $\mathrm{X}$ agrupada com a região I (SANTOS et al., 2018).

Para determinar os SCC no clima futuro foram utilizadas as projeções climáticas do Couple Model Intercomparison Project Phase 5 (CMIP5, TAYLOR et al., 2012). Consideraram-se três períodos: $P 1=2011-2040, P 2=2041-2070$ e $\mathrm{P} 3=2071-2100$. As variáveis utilizadas foram médias mensais de temperatura do ar próxima à superfície e acumulado mensal de precipitação. Esses dados correspondem à média (ensemble) de 24 modelos de projeção climática do CMIP5, disponibilizados no Earth System Grid data portal (TAYLOR et al., 2012). Os 24 modelos utilizados no ensemble foram: ACCES 1.0, BCC-CSM1-1, CanESM2, CCSM4, CNRMC-M5, CSIRO-Mk3-6-0, EC-EARTH, FGOALS-g2, FIOESM, GFDL-CM3， GFDL-ESM2G， GFDL-ESM2M， Giss-E2-R, HadGEM2-CC, HadGEM2-ES, INMCM4, IPSL-CM5A-LR, IPSL-CM5A-MR, MIROC-ESM, MIROCESM-CHEM, MIROC5, MPI-ESM-LR, MRI-CGCM3 e NorESM1-M. Como a resolução horizontal dos 24 modelos varia entre 1 a 3 graus de latitude/longitude, para computar a média de uma dada variável entre esses modelos, todas as variáveis foram previamente interpoladas (com o método bilinear) para uma grade regular com $1^{\circ} \times 1^{\circ}$ de latitude/longitude.

Analisaram-se quatro cenários de mudanças climáticas do Representative Concentration Pathways (RCPs) 2.6, 4.5, 6.0 e 8.5; sendo o cenário RCP 2.60 que apresenta a menor forçante radiativa $\left(2.6 \mathrm{~W} \mathrm{~m}^{-2}\right)$ e o RCP8.5 o que apresenta a maior forçante radiativa $\left(8.5 \mathrm{~W} \mathrm{~m}^{-2}\right.$ ) (VAN VUUREN et al., 2011).

Tabela 1 - Cidades, região de planejamento correspondente, localização e número da estação meteorológica utilizada nesse estudo.

\begin{tabular}{lcclcc}
\hline Cidades & Região & Estação & Cidades & Região & Estação \\
\hline Aimorés & X & 1 & Joao Pinheiro & VII & 26 \\
Araxá & V & 2 & Juiz de Fora & II & 27 \\
Arinos & VII & 3 & Lambari & III & 28 \\
Bambuí & VI & 4 & Lavras & III & 29 \\
Barbacena & I & 5 & Machado & III & 30 \\
Belo Horizonte & I & 6 & Maria da Fé & III & 31 \\
Bom Despacho & VI & 7 & Moçambinho & VIII & 32 \\
Conceição do Mato Dentro & I & 8 & Monte Azul & VIII & 33 \\
Caparão & II & 9 & Montes Claros & VIII & 34 \\
Capinópolis & IV & 10 & Oliveira & VI & 35 \\
Caratinga & X & 11 & Paracatu & VII & 36 \\
Carbonita & IX & 12 & Passa Quatro & III & 37 \\
Coronel Pacheco & II & 13 & Patos de Minas & V & 38 \\
Curvelo & I & 14 & Pedra Azul & IX & 39 \\
Diamantina & I & 15 & Pirapora & VIII & 40 \\
Divinópolis & VI & 16 & Poços de Caldas & III & 41 \\
Espinosa & VIII & 17 & Pompeu & I & 42 \\
Florestal & I & 18 & Salinas & VIII & 43 \\
Formoso & VII & 19 & São Lourenço & III & 44 \\
\hline
\end{tabular}




\begin{tabular}{lcllcc}
\hline Frutal & IV & 20 & São Sebastião do Paraíso & III & 45 \\
Ibirité & I & 21 & Sete Lagoas & I & 46 \\
Itamarandiba & IX & 22 & Uberaba & IV & 47 \\
Ituiutaba & IV & 23 & Unaí & VII & 48 \\
Januária & VIII & 24 & Viçosa & II & 49 \\
João Monlevade & I & 25 & & & \\
\hline
\end{tabular}

As simulações no clima presente, em geral, quando comparadas com as observações (dados medidos nas estações meteorológicas) apresentam viés (bias), isto é, diferenças. Isso ocorre uma vez que os modelos são aproximações da realidade. Para reduzir a diferença entre simulação e dado observado podese empregar diferentes técnicas de correção de bias. A técnica empregada no presente estudo foi (REBOITA et al 2017): (a) a média climatológica do período presente dos pontos de grade da simulação mais próximos aos das 49 estações meteorológicas foi comparada à média dessas e calculada a diferença entre elas (simulação menos observação) e (b) o valor obtido foi somado ou subtraído tanto das simulações do período presente quando do futuro (P1, P2 e P3).

O SCC de Thornthwaite requer dados de deficiência hídrica (DEF) e excedente hídrico (EXC) oriundos do extrato do BHC, ambos em milímetros $(\mathrm{mm})$. Neste estudo, utilizou para o cálculo do BHC o método proposto por Thornthwaite e Mather (1957) simplificado por Pereira (2005). Para o cálculo do BHC, além das médias climatológicas mensais de precipitação, foram utilizadas as médias climatológicas mensais de temperatura na estimativa de evapotranspiração potencial (ETP). O método utilizado para a estimativa de ETP foi o de Thornthwaite (1948); uma vez que apresenta bons resultados para MG (SANTOS et al., 2017), além de ser o método de estimativa de ETP mais utilizado para estudos de BHC (SENTELHAS et al., 2008). Foi considerado no BHC a capacidade de água disponível (CAD) de $100 \mathrm{~mm}$, por ser um valor recomendado em estudos climatológicos (SENTELHAS et al., 2008; VIANELLO; ALVES, 2012).

\subsection{SCC DE KÖPPEN}

De posse dos dados médios mensais de precipitação $(\mathrm{mm})$ e temperatura média do ar $\left({ }^{\circ} \mathrm{C}\right)$, obtida pela média aritmética das temperaturas mínimas e máximas, determinou-se o SCC de Köppen, seguindo as modificações propostas por Alvares et al. (2014). Tais modificações consistem no maior detalhamento dos tipos climáticos em relação à metodologia usada, por exemplo, em Reboita et al. (2015). No SCC de Köppen, os tipos climáticos são simbolizados por duas ou três letras (Tabela 2). A primeira letra indica a zona climática, sendo definida pela temperatura do mês mais frio e precipitação. A segunda considera a distribuição sazonal da precipitação e a terceira considera a variação sazonal da temperatura do mês mais quente. Neste estudo, manteve-se o valor de referência para a precipitação do mês mais seco (PSECO) de $60 \mathrm{~mm}$ similar a Reboita et al. (2015), e não como o proposto por Alvares et al. (2014) de 40 $\mathrm{mm}$. Isso evita que áreas sejam erroneamente classificadas como sem estação seca definida, pois a climatologia de MG mostra totais médios no inverno entre 40 a 60 mm (GARCIA et al., 2018). 
Para o grupo B (árido, semiárido e desértico), o limiar da precipitação (Plimiar) foi obtido a partir da temperatura média anual (Ta) $\left({ }^{\circ} \mathrm{C}\right)$ e da precipitação acumulada anual $(\mathrm{Pa})\left(\mathrm{mm} \mathrm{ano}^{-1}\right)$, dada pela Eq. 1 (KOTTEK et al., 2006):

$P_{\text {limiar }}=\left\{\begin{array}{l}2 T a, \text { Pelo menos } 2 / 3 \text { da Pa ocorre no inverno } \\ (2 T a)+28, \text { Pelo menos } 2 / 3 \text { da Pa ocorre no verão } \\ (2 T a)+14, \text { Caso contrário }\end{array}\right.$ 
Tabela 2 - Critérios para a classificação climática de Köppen baseado na simplificação realizada por Alvares et al. (2014).

\begin{tabular}{|c|c|c|c|c|c|c|c|c|}
\hline \multicolumn{3}{|c|}{ Temperatura } & \multicolumn{2}{|c|}{ Precipitação } & \multirow{2}{*}{\multicolumn{3}{|c|}{ Clima }} & \multirow{3}{*}{\begin{tabular}{|c|} 
Símbolo \\
$\mathrm{Af}$ \\
\end{tabular}} \\
\hline$T_{\text {FRIO }}$ & Touente & Ta & & $\mathbf{P a}$ & & & & \\
\hline \multirow{8}{*}{$\geq 18^{\circ} \mathrm{C}$} & & & $\mathrm{P}_{\mathrm{SECO}} \geq 60 \mathrm{~mm}$ & & \multirow{4}{*}{$\begin{array}{c}\text { A } \\
\text { (megatérmico } \\
\text { ou tropical) }\end{array}$} & & f (sem estação seca definida) & \\
\hline & & & \multirow{7}{*}{$\mathrm{P}_{\mathrm{SECO}}<60 \mathrm{~mm}$} & $\geq 25\left(100-P_{\text {SECO }}\right)$ & & & m (monçônico) & $\mathrm{Am}$ \\
\hline & & & & $<25(100-$ PVSECO$)$ & & & s (com seca no verão) & As \\
\hline & & & & $<25\left(100-\right.$ PISECO $_{\text {IS }}$ & & & w (com seca no inverno) & Aw \\
\hline & & $\stackrel{\geq}{18^{\circ} \mathrm{C}}$ & & $\geq 5 * P_{\text {LIMIAR }}$ & \multirow{4}{*}{$\begin{array}{c}\mathrm{B} \\
\text { (árido) }\end{array}$} & \multirow{2}{*}{ (semiárido ou estepe) } & h (baixa latitude e altitude) & BSh \\
\hline & & $\begin{array}{c}< \\
18^{\circ} \mathrm{C}\end{array}$ & & $<10 * P_{\text {LIMIAR }}$ & & & $\begin{array}{c}\text { k (média latitude e alta } \\
\text { altitude) }\end{array}$ & BSk \\
\hline & & $\begin{array}{c}\geq \\
18^{\circ} \mathrm{C}\end{array}$ & & $<5 * P_{\text {LIMIAR }}$ & & \multirow{2}{*}{ W (árido ou desértico) } & h (baixa latitude e altitude) & BWh \\
\hline & & $18^{\circ} \mathrm{C}$ & & & & & $\begin{array}{c}\text { k (média latitude e alta } \\
\text { altitude) }\end{array}$ & BWk \\
\hline \multirow[t]{2}{*}{$\begin{array}{r}\geq-3^{\circ} \mathrm{C} \\
\mathrm{e}<18^{\circ} \mathrm{C}\end{array}$} & $\geq 22^{\circ} \mathrm{C}$ & & $\mathrm{P}_{\mathrm{SECO}}>60 \mathrm{~mm}$ & & \multirow{9}{*}{$\begin{array}{c}\text { C } \\
\text { (mesotérmico } \\
\text { ou temperado } \\
\text { quente ou } \\
\text { subtropical) }\end{array}$} & \multirow{3}{*}{$\begin{array}{c}f \\
\text { (clima oceânico; sem } \\
\text { estação seca definida) }\end{array}$} & a (com verão quente) & Cfa \\
\hline & $\begin{array}{l}<22^{\circ} \mathrm{Ce} \\
\mathrm{T}_{\mathrm{M} 10} \geq 4\end{array}$ & & & & & & b (com verão temperado) & $\mathrm{Cfb}$ \\
\hline $\begin{array}{l}\geq-38^{\circ} \mathrm{C} \\
\mathrm{e}<18^{\circ} \mathrm{C}\end{array}$ & $\begin{array}{l}<22^{\circ} \mathrm{C} \text { e } 1 \\
\leq \mathrm{T}_{\mathrm{M} 10}<4\end{array}$ & & & & & & c (com verão curto e frio) & Cfc \\
\hline \multirow[t]{2}{*}{$\begin{array}{l}\geq-3^{\circ} \mathrm{C} \\
\mathrm{e} 18^{\circ} \mathrm{C}\end{array}$} & $\geq 22^{\circ} \mathrm{C}$ & & $\mathrm{P}_{\mathrm{SECO}}<60 \mathrm{~mm}$ & & & \multirow{3}{*}{$\begin{array}{c}\mathrm{w} \\
\text { (com seca no inverno) }\end{array}$} & a (e verão quente) & Cwa \\
\hline & $\begin{array}{l}<22^{\circ} \mathrm{Ce} \\
\mathrm{T}_{\mathrm{M} 10} \geq 4\end{array}$ & & $\begin{array}{l}\text { PVUMIDO } \geq \\
10 * P_{\text {ISECO }}\end{array}$ & & & & b (e verão temperado) & Cwb \\
\hline $\begin{array}{l}\geq-38^{\circ} \mathrm{C} \\
\mathrm{e}<18^{\circ} \mathrm{C}\end{array}$ & $\begin{array}{l}<22^{\circ} \mathrm{C} \text { e } 1 \\
\leq \mathrm{T}_{\mathrm{M} 10}<4\end{array}$ & & & & & & c (e verão curto e frio) & Cwc \\
\hline \multirow[t]{2}{*}{$\begin{array}{c}\geq-3^{\circ} \mathrm{C} \\
<e< \\
18^{\circ} \mathrm{C}\end{array}$} & $\geq 22^{\circ} \mathrm{C}$ & & $\mathrm{P}_{\mathrm{SECO}}<60 \mathrm{~mm}$ & & & \multirow{3}{*}{$\begin{array}{c}\text { s } \\
\text { (com seca no verão) }\end{array}$} & a (e quente) & Csa \\
\hline & $\begin{array}{l}<22^{\circ} \mathrm{Ce} \\
\mathrm{T}_{\mathrm{M} 10} \geq 4\end{array}$ & & $\begin{array}{l}\text { PIUMIDO } \geq \\
3 * P_{\text {VSECO }}\end{array}$ & & & & b (e temperado) & Csb \\
\hline $\begin{array}{l}\geq-38^{\circ} \mathrm{C} \\
\mathrm{e}<18^{\circ} \mathrm{C}\end{array}$ & $\begin{array}{l}<22^{\circ} \mathrm{C} \text { e } 1 \\
\leq \mathrm{T}_{M 10}<4\end{array}$ & & $\begin{array}{l}\text { PVUMIDo } \geq \\
10 * P_{\text {ISECO }}\end{array}$ & & & & c (e verão curto e frio) & Csc \\
\hline$\geq-38^{\circ} \mathrm{C}$ & $\geq 22^{\circ} \mathrm{C}$ & & $\mathrm{P}_{\mathrm{SECO}}>60 \mathrm{~mm}$ & & D & $\mathrm{f}$ & a (com verão quente) & Dfa \\
\hline
\end{tabular}




\begin{tabular}{|c|c|c|c|c|c|c|}
\hline \multirow[t]{3}{*}{$\mathrm{e}<-3^{\circ} \mathrm{C}$} & & & \multirow{12}{*}{$\begin{array}{c}\text { (microtérmico } \\
\text { ou temperado } \\
\text { frio ou } \\
\text { continental) }\end{array}$} & \multirow{4}{*}{$\begin{array}{c}\text { (sem estão seca } \\
\text { definida) }\end{array}$} & & \\
\hline & $\begin{array}{l}<22^{\circ} \mathrm{Ce} \\
\mathrm{T}_{\mathrm{M} 10} \geq 4\end{array}$ & & & & b (com verão temperado) & Dfb \\
\hline & $\begin{array}{l}<22^{\circ} \mathrm{C} \text { e } 1 \\
\leq \mathrm{T}_{\mathrm{M} 10}<4\end{array}$ & & & & c (com verão curto e frio) & Dfc \\
\hline $\begin{array}{l}<-38^{\circ} \mathrm{C} \\
\mathrm{e}<-3^{\circ} \mathrm{C}\end{array}$ & & & & & $\mathrm{d}$ (com inverno muito frio) & Dfd \\
\hline \multirow[t]{3}{*}{$\begin{array}{l}\geq-38^{\circ} \mathrm{C} \\
\mathrm{e}<-3^{\circ} \mathrm{C}\end{array}$} & $\geq 22^{\circ} \mathrm{C}$ & $\mathrm{P}_{\mathrm{SECO}}<60 \mathrm{~mm}$ & & \multirow{4}{*}{$\begin{array}{c}\text { w } \\
\text { (com seca no inverno) }\end{array}$} & a (com verão quente) & Dwa \\
\hline & $\begin{array}{l}<22^{\circ} \mathrm{C} \mathrm{e} \\
\mathrm{T}_{\mathrm{M} 10} \geq 4\end{array}$ & $\begin{array}{l}\text { PVUMIDO } \geq \\
10 * P_{\text {ISECO }}\end{array}$ & & & b (e verão temperado) & Dwb \\
\hline & $\begin{array}{l}<22^{\circ} \mathrm{C} \text { e } 1 \\
\leq T_{M 10}<4\end{array}$ & & & & c (e verão curto e frio) & Dwc \\
\hline $\begin{array}{l}<-38^{\circ} \mathrm{C} \\
\mathrm{e}<-3^{\circ} \mathrm{C}\end{array}$ & & & & & $\mathrm{d}$ (e inverno muito frio) & Dwd \\
\hline \multirow[t]{3}{*}{$\begin{array}{l}\geq-38^{\circ} \mathrm{C} \\
\mathrm{e}<-3^{\circ} \mathrm{C}\end{array}$} & $\geq 22^{\circ} \mathrm{C}$ & $\mathrm{P}_{\mathrm{SECO}}<60 \mathrm{~mm}$ & & \multirow{4}{*}{ (com seca no verão) } & a (e quente) & Dsa \\
\hline & $\begin{array}{l}<22^{\circ} \mathrm{C} \mathrm{e} \\
\mathrm{T}_{\mathrm{M} 10} \geq 4\end{array}$ & $\begin{array}{l}\text { PIUMIDO }_{\text {I }} \geq \\
3 * P_{V S E C O}\end{array}$ & & & b (e temperado) & Dsb \\
\hline & $\begin{array}{l}<22^{\circ} \mathrm{C} \text { e } 1 \\
\leq \mathrm{T}_{\mathrm{M} 10}<4\end{array}$ & $\begin{array}{l}\text { PVUMIDO } \geq \\
10 * P_{\text {ISECO }} \\
\end{array}$ & & & c (e verão curto e frio) & Dsc \\
\hline \multirow[t]{3}{*}{$\begin{array}{l}<-38^{\circ} \mathrm{C} \\
\mathrm{e}<-3^{\circ} \mathrm{C}\end{array}$} & & & & & d (e inverno muito frio) & Dsd \\
\hline & $\begin{array}{c}<10 \text { e } \geq \\
0^{\circ} \mathrm{C}\end{array}$ & & \multirow{2}{*}{$\begin{array}{c}\text { E } \\
\text { (glacial ou } \\
\text { polar) }\end{array}$} & & $\mathrm{T}$ (tundra) & ET \\
\hline & $<0^{\circ} \mathrm{C}$ & & & & $\mathrm{F}$ (polar) & $\mathrm{EF}$ \\
\hline
\end{tabular}

$\mathrm{T}_{\text {FRIO }}=$ temperatura do mês mais frio; $\mathrm{T}_{\text {QUENTE }}=$ temperatura do mês mais quente; Ta $=$ temperatura média anual; $\mathrm{P}_{\mathrm{M}}=$ precipitação mensal; $\mathrm{Pa}=$ precipitação acumulada anual; $\mathrm{P}_{\mathrm{SECO}}=$ precipitação do mês mais seco; $\mathrm{PVSECO}_{\mathrm{V}}$ = precipitação do mês mais seco no verão; $\mathrm{P}_{\mathrm{ISECO}}$ = precipitação do mês mais seco no inverno; Pvumido = precipitação do mês mais úmido no verão; PIUmido = precipitação do mês mais úmido no inverno; $\mathrm{T}_{\mathrm{M} 10}=$ número de meses em que a temperatura é superior a $10^{\circ} \mathrm{C} ; \mathrm{P}_{\text {LIMIAR }}=$ varia de acordo com a temperatura média anual e a sazonalidade da precipitação, segundo equação 12. Para MG, o verão é definido pelo período de seis meses mais quentes ( $S, O, N, D, J, F)$ e o inverno pelo período de seis meses mais frios ( $M, A, M, J, J, A)$; PSECO $=60 \mathrm{~mm}$ foi baseado em Reboita et al. (2015). 


\subsection{SCC DE THORNTHWAITE}

Para o SCC de Thornthwaite foi utilizada a metodologia de Thornthwaite adaptada por Camargo (1999), o qual baseia-se em índices calculados dos valores extraídos do extrato do BHC: índice hídrico (Ih), índice de aridez (Ia) e índice de umidade (Iu) (Eqs. 2, 3 e 4). A metodologia detalhada do cálculo do BHC pode ser encontrada em Pereira (2005) e Santos (2018). Para o cômputo dos índices são necessários o EXC anual $\left(\mathrm{mm}^{\mathrm{ano}}{ }^{-1}\right)$, a DEF anual $\left(\mathrm{mm} \mathrm{ano}^{-1}\right) \mathrm{e}$ a ETP anual ( $\left.\mathrm{mm}^{\mathrm{ano}}{ }^{-1}\right)$. Esses índices são utilizados na determinação dos tipos (primeira letra da classificação) e subtipos climáticos (segunda letra da classificação) para o fator hídrico (Tabela 3) e fator térmico (Tabela 4). Para o fator hídrico, o tipo climático é representado pelo Iu, enquanto que o subtipo climático é determinado pelo Ia ou Ih (Tabela 3 ).

Ih $=($ EXC anual/ETPanual $) * 1$

$\mathrm{Ia}=($ DEF anual $/$ ETPanual $) * 100$

$\mathrm{Iu}=\mathrm{Ih}-0,6 * \mathrm{Ia}$

Tabela 3 - Tipos climáticos e subtipos do fator hídrico da classificação climática de Thornthwaite.

\begin{tabular}{|c|c|c|}
\hline Tipo climático hídrico & Símbolo & Classificação \\
\hline Superúmido & A & Iu $\geq 100$ \\
\hline Úmido & B4 & $80 \leq \mathrm{Iu}<100$ \\
\hline Úmido & B3 & $60 \leq \mathrm{Iu}<80$ \\
\hline Úmido & B2 & $40 \leq \mathrm{Iu}<60$ \\
\hline Úmido & B1 & $20 \leq \mathrm{Iu}<40$ \\
\hline Subúmido & $\mathrm{C} 2$ & $0 \leq \mathrm{Iu}<20$ \\
\hline Subúmido seco & $\mathrm{C} 1$ & $-20 \leq \mathrm{Iu}<0$ \\
\hline Semiárido & $\mathrm{D}$ & $-40 \leq$ Iu $<-20$ \\
\hline Árido & $E$ & $-60 \leq$ Iu $<-40$ \\
\hline \multicolumn{3}{|c|}{ Para climas úmidos $(A, B, C 2)$ - utilizar o Ia } \\
\hline Subtipo climático hídrico & Símbolo & Índice de aridez (Ia) \\
\hline Sem ou com pequena deficiência hídrica & $\mathrm{r}$ & $0 \leq$ Ia $<16,7$ \\
\hline Deficiência hídrica moderada no verão & $\mathrm{s}$ & $16,7 \leq$ Ia $<33,3$ \\
\hline Deficiência hídrica moderada no inverno & w & $16,7 \leq$ Ia $<33,3$ \\
\hline Grande deficiência hídrica no verão & s2 & Ia $\geq 33,3$ \\
\hline Grande deficiência hídrica no inverno & w2 & Ia $\geq 33,3$ \\
\hline \multicolumn{3}{|c|}{ Para climas secos $(\mathrm{C} 1, \mathrm{D}$ E) - utilizar o Ih } \\
\hline Subtipo climático hídrico & Símbolo & Índice hídrico (Ih) \\
\hline Excedente hídrico pequeno ou nulo & $\mathrm{d}$ & $0 \leq$ Ih $<10$ \\
\hline Excedente hídrico moderado no verão & s & $10 \leq$ Ih $<20$ \\
\hline Excedente hídrico moderado no inverno & w & $10 \leq \mathrm{Ih}<20$ \\
\hline Grande excedente hídrico no verão & s2 & Ih $\geq 20$ \\
\hline Grande excedente hídrico no inverno & w2 & Ih $\geq 20$ \\
\hline
\end{tabular}


Para o fator térmico, os tipos climáticos foram determinados a partir da ETP e os subtipos climáticos são baseados na relação entre somatório da ETP nos meses do verão (ETPverão) e a ETP anual (Tabela 4):

$\mathrm{SCT}=\frac{\text { ETP }_{\text {Verão }}}{\text { ETPanual }} \times 10$

Tabela 4 - Tipos e subtipos climáticos para o fator térmico da classificação climática de Thornthwaite, baseado na evapotranspiração potencial (ETP).

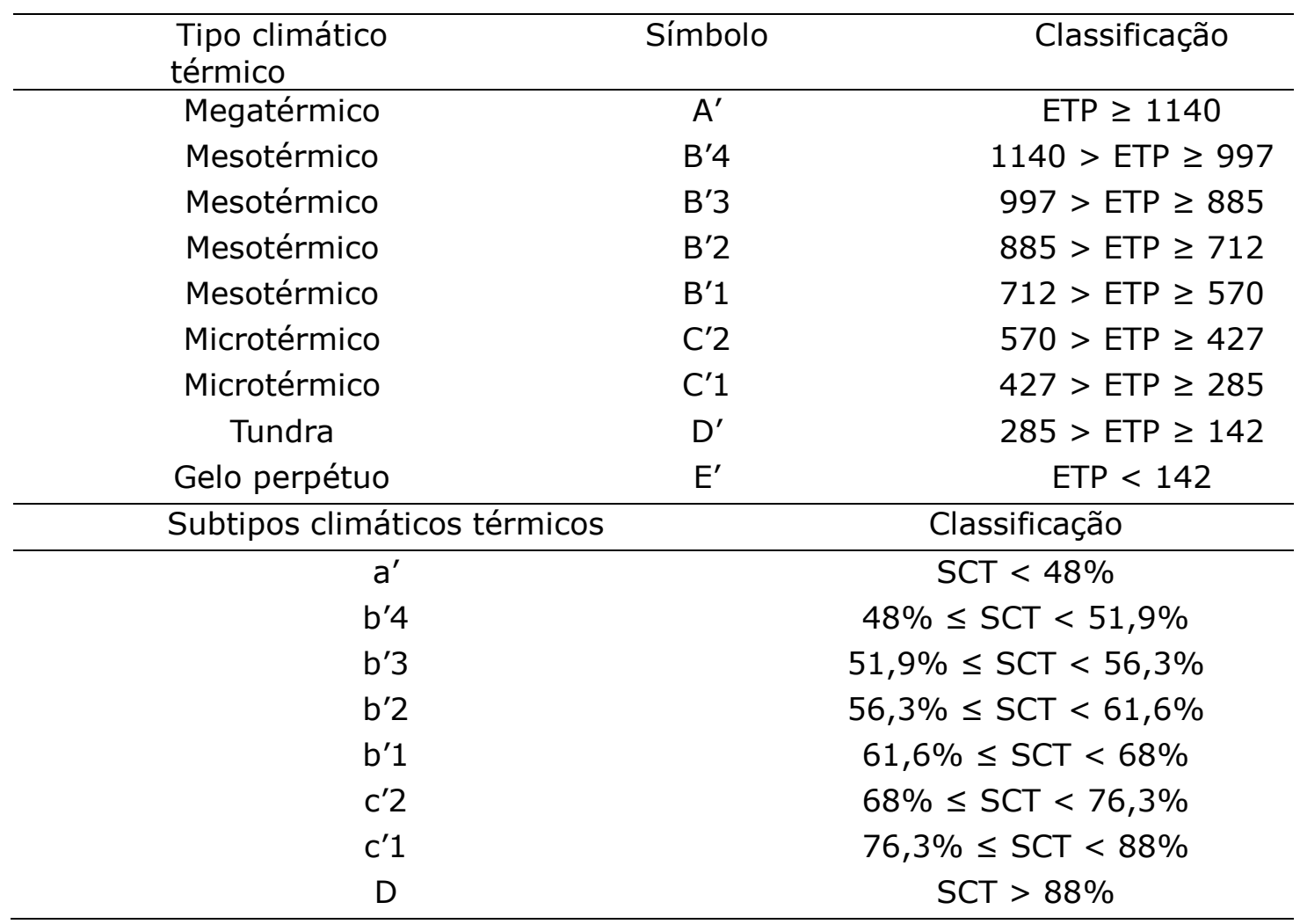

Uma vez calculados os SCC para o clima presente (1981 a 2010) e futuro ( $P 1=2011$ a 2040, P2=2041 a 2070, P3=2071 a 2100), tais informações foram interpoladas para resolução horizontal de $0.1^{\circ} \times 0.1^{\circ}$ a fim de melhorar a apresentação visual nos mapas de MG. Para o clima presente são apresentados apenas os mapas obtidos com os dados das estações meteorológicas e, para o futuro, através da média (ensemble) das projeções dos modelos climáticos.

Os dados dos SCC foram submetidos à análise de variância (ANOVA) seguida de comparação de médias pelo teste Tukey a $5 \%$ (STORCK et al., 2011), a fim de verificar se há diferenças dos índices climáticos obtidos entre cada SCC no clima presente (dados das estações meteorológicas) e futuro (projeções em diferentes períodos e RCPs). Para proceder a ANOVA, os dados foram analisados segundo um delineamento em blocos ao acaso, organizado em esquema fatorial $(49 \times 4)$ com três blocos por tratamento. Cada tratamento constituiu da combinação dos 49 níveis do fator A (estações meteorológicas) e os 4 níveis do fator $D$ (RCPs 2.6, 4.5, 6.0 e 8.5). Consideraram-se como blocos 
as três projeções, sendo P1 (2011-2040), P2 (2041-2070) e P3 (2071-2100). Os testes foram realizados através do software SISVARß versão 5.6 (FERREIRA, 2011).

\section{RESULTADOS E DISCUSSÃO}

\subsection{CLASSIFICAÇÃO CLIMÁTICA DE KÖPPEN}

Segundo a classificação climática de Köppen, considerando o clima presente (1981-2010), MG apresenta cinco tipos climáticos (Figura 2), sendo dois temperados quentes (Cwb e Cwa), um tropical (Aw) e dois áridos (BSh e BWh), corroborando com o estudo de Reboita et al. (2015) e diferente dos estudos de Sá Junior (2009) e Ávila et al. (2014), que indicaram a ocorrência dos climas Cwa, Cwb, BSh e Aw, mas não observaram a ocorrência do clima BWh, além de observarem a ocorrência do BSw.

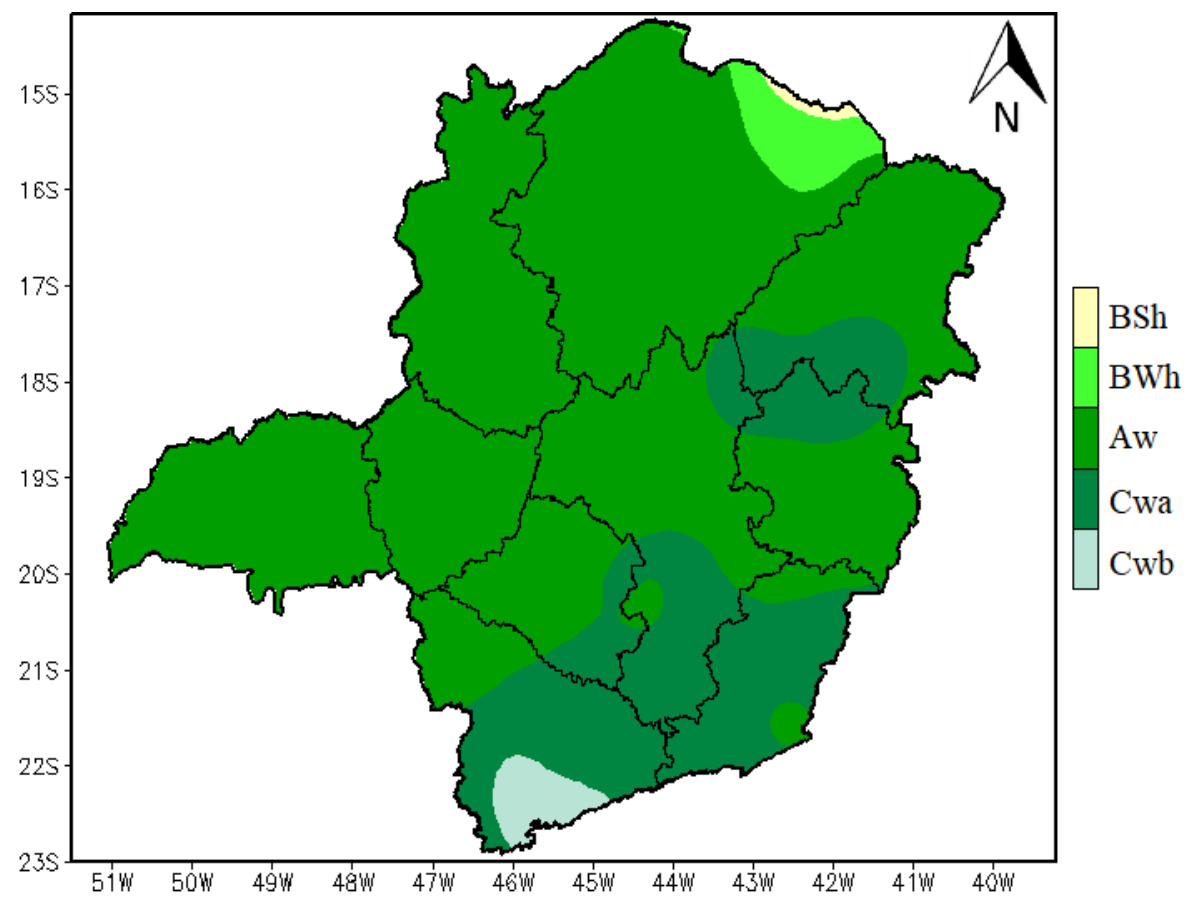

Figura 2 - Classificação climática de Köppen para Minas Gerais considerando o clima presente (1981-2010).

No clima presente, a predominância é dos climas Aw e Cwa, tropical e temperado quente, ambos com período seco no inverno (Figura 2). O clima Aw ocorre nas regiões Central, Triângulo, Alto Parnaíba, Centro-oeste, Noroeste, Norte, Jequitinhonha e Rio Doce (Regiões I, IV, V, VI, VII, VIII, IX e X; respectivamente), enquanto que o clima Cwa prevalece nas regiões da Mata e Sul (Regiões II e III, respectivamente). O clima mineiro é fortemente influenciado pelo relevo, o que contribui para a formação de microclimas dentro de mesmas regiões (ÁVILA et al., 2014). A região Sul (região III), por exemplo, conhecida por suas montanhas, apresentou nas cidades de maior altitude (Maria da Fé e Poços de Caldas com 1276,33 m e $1150 \mathrm{~m}$ de altitude, respectivamente) clima com temperatura mais amena (Cwb), enquanto a cidade de menor altitude 
(São Sebastião do Paraíso com 820 m de altitude) apresentou clima com temperaturas mais elevadas (Aw). Essa diferença de tipos climáticos em MG, considerando o clima presente, ocorre devido à variação espacial e temporal da precipitação, que é influenciada pelo sistema de Monção Sul-Americana (MSA) (REBOITA et al., 2015), de forma que o inverno é seco e o verão é chuvoso. Com relação à variação espacial das temperaturas, essa ocorre principalmente por influência das diferentes altitudes da topografia. Além disso, a entrada de frentes frias, principalmente na porção sul de $M G$, reduz as temperaturas no inverno e atua na ocorrência de clima temperado (Cwa e Cwb) (GARCIA et al., 2018).

Com relação ao clima futuro ( $\mathrm{P} 1, \mathrm{P} 2$ e $\mathrm{P} 3)$, são projetadas mudanças no SCC de Köppen (Figura 3). De maneira geral, o aumento projetado na temperatura do ar, chegando a $5^{\circ} \mathrm{C}$ (P3) para algumas localidades de MG (SANTOS et al., 2017), impactará no aumento da prevalência de climas mais quentes, mesmo no cenário mais brando (RCP2.6) (Figura 3a). O aumento da irregularidade das chuvas em Minas Gerais (SANTOS et al., 2017) também deve afetar diretamente no aparecimento, assim como no aumento, de climas BSh e BWh (áridos), principalmente nas regiões Norte e dos Vales de Jequitinhonha e Mucuri (Regiões VIII e IX, respectivamente) nos RCP's 2.6, 4.5, 6.0 e 8.5 (Figura 3).

Do clima presente (Figura 2) para o P1 (2011-2040) (Figura 3), há aumento considerável de áreas classificadas como Aw (quente), redução das áreas classificadas, principalmente, como Cwb (temperaturas mais brandas), além do surgimento de áreas de clima BSh (semiárido característico de estepe). No P2 (2041-2070) e P3 (2071-2100), exceto para RCP 2.6, há crescimento das áreas classificadas como $\mathrm{BWh}$ e $\mathrm{BSh}$, e redução significativa das áreas classificadas como Cwb e Cwa (Figura 3a, 3b e 3c). 


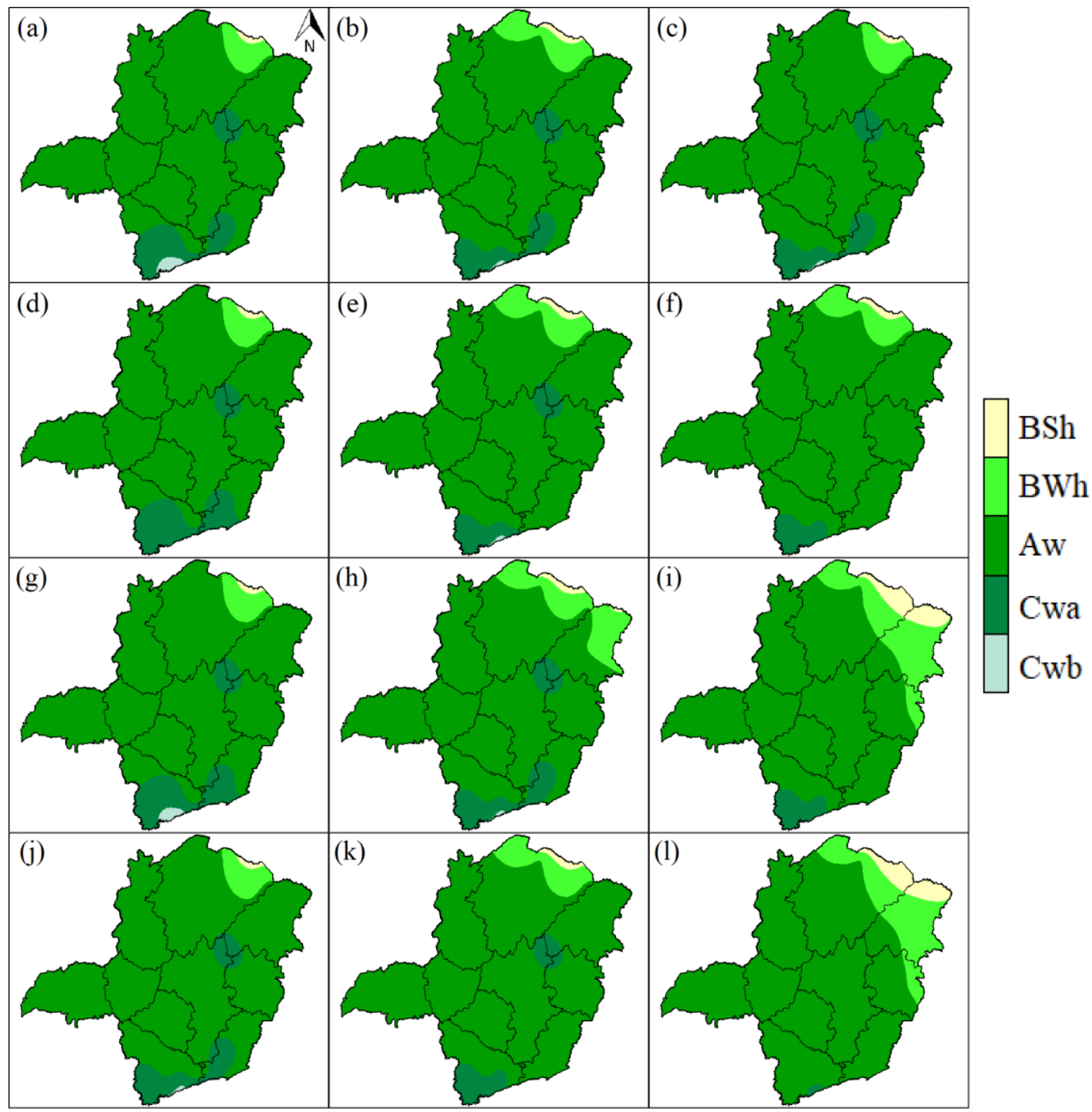

Figura 3 - Distribuição geográfica da classificação climática de Köppen para o estado de Minas Gerais: a) cenário RCP 2.6 para o período $\mathrm{P} 1$; b) cenário RCP 2.6 para o período $\mathrm{P} 2$; c) cenário RCP 2.6 para o período $\mathrm{P} 3$; d) cenário RCP 4.5 para o período $\mathrm{P} 1$; e) cenário RCP 4.5 para o período $\mathrm{P} 2$; f) f cenário RCP 4.5 para o período $\mathrm{P} 3$; g) cenário RCP 6.0 para o período $\mathrm{P} 1$; h) cenário RCP 6.0 para o período $\mathrm{P} 2$; i) cenário RCP 6.0 para o período $P 3 ; j$ ) cenário RCP 8.5 para o período $P 1 ; k$ ) cenário RCP 8.5 para o período P2; I) cenário RCP 8.5 para o período P3.

Pela ANOVA (Tabela 5), foi possível verificar o comportamento da classificação climática ao longo das projeções (blocos) para cada estação meteorológica (FATOR A) e RCP (FATOR D). Observou-se que as regiões Central, Sul, Triângulo, Alto Parnaíba, Centro-Oeste, Noroeste de Minas, Norte de Minas e Rio Doce (Regiões I, III, IV, V, VI, VII, VII e X, respectivamente) apresentam variância não significativa entre as classificações climáticas entre os RCP's e projeções. Isto significa que não há mudança das classificações climáticas, ou as mudanças são praticamente desprezíveis ao longo das projeções. Como exemplo, nas regiões Central e Rio Doce, as cidades de Belo 
Horizonte, Conceição do Mato Dentro, Curvelo, João Monlevade, Pompeu, Sete Lagoas e Caratinga (Estações 6, 8, 14, 25, 42, 46 e 11; respectivamente) apresentam classificação Aw para o clima presente e mantém essa classificação até a P3 no RCP 8.5. Por outro lado, as cidades de Florestal e Ibirité (Estações 18 e 21 , respectivamente) apresentam modificação na classificação climática de Cwa (temperado quente) para Aw (tropical) já no P1 e no RCP 2.6, enfatizado pelo aumento projetado de temperatura do ar (SANTOS et al., 2017) (Tabela 5) e essa classificação se mantém constante até o P3. Diamantina e Aimorés (Estações 15 e 1; respectivamente) apresentam padrão análogo ao de Florestal e Ibirité, isto é, prevalência de clima mais quente e seco, porém, essa mudança só é projetada para o final do século XXI (P3).

Ressalta-se que as regiões do Triângulo Mineiro, do Alto Parnaíba, e Norte de Minas não apresentam diferença no SCC de Köppen independentemente da estação meteorológica, cenário climático (RCP) e projeção (PR1, PR2 3 e PR3), não sendo possível aplicar a ANOVA para estas regiões. Isso significa que para as três regiões (IV, V e VII) há prevalência do clima Aw no clima presente até final do século XXI.

Para as regiões da Zona da Mata e Vales do Jequitinhonha/Mucuri (Regiões II e IX, respectivamente), a variância é significativa entre as classificações, com tendência análoga às demais regiões com intensificação da temperatura do ar e redução da precipitação. Como exemplo, na região da Mata houve tendência de alteração de climas classificados como Cwa (clima presente) para Aw já na P1, e no Vales do Jequitinhonha/Mucuri como exemplo Pedra Azul, tendência de alteração de clima classificado como Aw (clima presente) para BWh. Diante do exposto, observou-se que a maioria das regiões apresentou variância não significativa devido à baixa sensibilidade do SCC de Köppen às variações climáticas locais. 
Tabela 5 - Análise de variância (ANOVA) e comparação de médias pelo teste de Tukey para a classificação climática de Köppen, considerando cada estação meteorológica, RCP's (2.6, 4.5, 6.0 e 8.5) para o clima presente (1981-2010) e projeções (P1=2011-2040, P2=2041-2070, P3=2071-2100).

\begin{tabular}{|c|c|c|c|c|c|c|c|c|c|c|c|c|c|c|c|c|c|c|c|c|}
\hline \multirow{4}{*}{ 余 } & \multirow{4}{*}{$\begin{array}{l}\text { FATOR A } \\
\text { CIDADE }\end{array}$} & & & & & & & & & & FATOR & & & & & & & & & \\
\hline & & \multirow{3}{*}{ ANOVA } & \multicolumn{2}{|c|}{ ATUAL } & \multicolumn{4}{|c|}{ RCP 2.6} & \multicolumn{4}{|c|}{ RCP 4.5} & \multicolumn{4}{|c|}{ RCP 6.0} & \multicolumn{4}{|c|}{ RCP 8.5} \\
\hline & & & \multirow{2}{*}{ BLOCO } & \multirow{2}{*}{$\mathrm{T}$} & \multicolumn{3}{|c|}{ BLOCO } & \multirow{2}{*}{ T } & \multicolumn{3}{|c|}{ BLOCO } & \multirow{2}{*}{$\mathrm{T}$} & \multicolumn{3}{|c|}{ BLOCO } & \multirow{2}{*}{$\mathrm{T}$} & \multicolumn{3}{|c|}{ BLOCO } & \multirow{2}{*}{$\mathrm{T}$} \\
\hline & & & & & $\mathrm{P} 1$ & P2 & P3 & & $\mathrm{P} 1$ & $\mathrm{P} 2$ & P3 & & $\mathrm{P} 1$ & $\mathrm{P} 2$ & P3 & & P1 & P2 & P3 & \\
\hline \multirow{12}{*}{$\begin{array}{l}\underset{0}{\infty} \\
\mapsto\end{array}$} & Barbacena & \multirow{12}{*}{$0,0716 * *$} & Cwa & - & Cwa & Cwa & Cwa & - & Cwa & $A w$ & Aw & - & Cwa & Cwa & $\mathrm{Aw}$ & - & Cwa & Aw & Aw & - \\
\hline & Belo Horizonte & & Aw & - & Aw & Aw & Aw & - & $A w$ & Aw & Aw & - & Aw & Aw & $A w$ & - & Aw & Aw & Aw & - \\
\hline & Conceição do Mato Dentro & & Aw & - & Aw & Aw & Aw & - & Aw & Aw & Aw & - & Aw & Aw & Aw & - & Aw & Aw & Aw & - \\
\hline & Curvelo & & $A w$ & - & Aw & Aw & Aw & - & Aw & $A w$ & Aw & - & Aw & Aw & Aw & - & Aw & Aw & Aw & - \\
\hline & Diamantina & & Cwa & - & Cwa & Cwa & Cwa & - & Cwa & Cwa & Aw & - & Cwa & Cwa & Aw & - & Cwa & Cwa & Aw & - \\
\hline & Florestal & & Cwa & - & Aw & Aw & Aw & - & Aw & Aw & Aw & - & Aw & Aw & Aw & - & Aw & Aw & Aw & - \\
\hline & Ibirité & & Cwa & - & Aw & Aw & Aw & - & Aw & Aw & Aw & - & Aw & Aw & Aw & - & Aw & Aw & Aw & - \\
\hline & João Monlevade & & Aw & - & Aw & Aw & $A w$ & - & Aw & $A w$ & Aw & - & Aw & Aw & Aw & - & Aw & Aw & Aw & - \\
\hline & Pompeu & & $A w$ & - & Aw & Aw & Aw & - & Aw & $A w$ & $A w$ & - & Aw & Aw & Aw & - & Aw & Aw & Aw & - \\
\hline & Sete Lagoas & & Aw & - & Aw & Aw & Aw & - & $A w$ & Aw & Aw & - & Aw & Aw & $A w$ & - & Aw & Aw & Aw & - \\
\hline & Aimorés & & Aw & - & Aw & Aw & Aw & - & Aw & Aw & Aw & - & Aw & Aw & BWh & - & Aw & Aw & BWh & - \\
\hline & Caratinga & & Aw & - & Aw & Aw & Aw & - & Aw & $A w$ & Aw & - & Aw & Aw & Aw & - & Aw & Aw & Aw & - \\
\hline \multirow{4}{*}{ ヨ } & Caparaó & \multirow{4}{*}{$0,0011^{*}$} & Cwa & $\mathrm{Aa}$ & Cwa & Cwa & Cwa & $\mathrm{Aa}$ & Cwa & Aw & Aw & $A b$ & Cwa & Aw & Aw & $A b$ & Cwa & Aw & Aw & $A b$ \\
\hline & Coronel Pacheco & & Aw & $\mathrm{Ba}$ & Aw & Aw & Aw & $\mathrm{Ba}$ & Aw & Aw & Aw & $\mathrm{Aa}$ & Aw & Aw & Aw & $\mathrm{Aa}$ & Aw & Aw & Aw & $\mathrm{Aa}$ \\
\hline & Juiz de Fora & & Cwa & $\mathrm{Aa}$ & Aw & Aw & Aw & $\mathrm{Bb}$ & Cwa & $A w$ & Aw & $A b$ & Cwa & Aw & Aw & $A b$ & Aw & Aw & Aw & $A b$ \\
\hline & Viçosa & & Cwa & $\mathrm{Aa}$ & Aw & Aw & Aw & $\mathrm{Bb}$ & Aw & Aw & Aw & $A b$ & Aw & Aw & Aw & $A b$ & Aw & Aw & Aw & $A b$ \\
\hline \multirow{2}{*}{ 㝍 } & Lambari & \multirow{2}{*}{$0,2111 * *$} & Cwa & - & Cwa & Cwa & Cwa & - & Cwa & Cwa & Cwa & - & Cwa & Cwa & Cwa & - & Cwa & Cwa & Aw & - \\
\hline & Lavras & & Cwa & - & Aw & Aw & Aw & - & Aw & $A w$ & $A w$ & - & Aw & Aw & Aw & - & Aw & Aw & Aw & - \\
\hline
\end{tabular}


Revista Brasileira de Climatologia

ISSN: 2237-8642 (Eletrônica)

\begin{tabular}{|c|c|c|c|c|c|c|c|c|c|c|c|c|c|c|c|c|c|c|c|c|}
\hline & Machado & & Cwa & - & Cwa & Aw & Aw & - & Cwa & Aw & Aw & - & Cwa & Aw & Aw & - & Aw & Aw & Aw & - \\
\hline & Maria da Fé & & Cwb & - & Cwb & Cwb & Cwb & - & Cwa & Cwb & Cwa & - & Cwb & Cwb & Cwa & - & Cwb & Cwa & Cwa & - \\
\hline & Passa Quatro & & Cwa & - & Cwa & Cwa & Cwa & - & Cwa & Cwa & Cwa & - & Cwa & Cwa & Cwa & - & Cwa & Aw & Aw & - \\
\hline & Poços de Caldas & & Cwb & - & Cwa & Cwa & Cwa & - & Cwa & Cwa & Cwa & - & Cwa & Cwa & Cwa & - & Cwa & Cwa & Aw & - \\
\hline & São Lourenço & & Cwa & - & Cwa & Cwa & Cwa & - & Cwa & Cwa & Aw & - & Cwa & Cwa & Aw & - & Cwa & Aw & Aw & - \\
\hline & São Sebastião do Paraíso & & Aw & - & Aw & Aw & Aw & - & Aw & Aw & Aw & - & Aw & Aw & Aw & - & Aw & Aw & Aw & - \\
\hline \multirow{6}{*}{$\begin{array}{l}> \\
0 \\
\geq\end{array}$} & Capinópolis & \multirow{6}{*}{$\begin{array}{c}\text { Todos as } \\
\text { classificações são } \\
\text { iguais, } \\
\text { independente do } \\
\text { cenário ou cidade }\end{array}$} & Aw & - & Aw & Aw & Aw & - & Aw & Aw & Aw & - & Aw & Aw & Aw & - & Aw & Aw & Aw & - \\
\hline & Frutal & & Aw & - & Aw & Aw & Aw & - & Aw & Aw & Aw & - & Aw & Aw & Aw & - & Aw & Aw & Aw & - \\
\hline & Ituiutaba & & Aw & - & Aw & Aw & Aw & - & Aw & Aw & $\mathrm{Aw}$ & - & Aw & Aw & Aw & - & Aw & Aw & Aw & - \\
\hline & Uberaba & & Aw & - & Aw & Aw & Aw & - & Aw & Aw & Aw & - & Aw & Aw & Aw & - & Aw & Aw & Aw & - \\
\hline & Araxá & & Aw & - & Aw & Aw & Aw & - & Aw & Aw & Aw & - & Aw & Aw & Aw & - & Aw & Aw & Aw & - \\
\hline & Patos de Minas & & Aw & - & Aw & Aw & Aw & - & Aw & Aw & Aw & - & Aw & Aw & Aw & - & Aw & Aw & Aw & - \\
\hline \multirow{4}{*}{$>$} & Bambuí & \multirow{4}{*}{$0,3349 * *$} & Cwa & - & Aw & Aw & Aw & - & Aw & Aw & Aw & - & Aw & Aw & Aw & - & Aw & Aw & Aw & - \\
\hline & Bom Despacho & & Aw & - & Aw & Aw & Aw & - & Aw & Aw & Aw & - & Aw & Aw & Aw & - & Aw & Aw & Aw & - \\
\hline & Divinópolis & & Aw & - & Aw & Aw & Aw & - & Aw & Aw & Aw & - & Aw & Aw & Aw & - & Aw & Aw & Aw & - \\
\hline & Oliveira & & Cwa & - & Aw & Aw & Aw & - & Aw & Aw & Aw & - & Aw & Aw & Aw & - & Aw & Aw & - & - \\
\hline \multirow{5}{*}{$\overrightarrow{5}$} & Arinos & \multirow{5}{*}{$\begin{array}{c}\text { Todos as } \\
\text { classificações são } \\
\text { iguais, } \\
\text { independente do } \\
\text { cenário ou cidade }\end{array}$} & Aw & - & Aw & Aw & Aw & - & Aw & Aw & Aw & - & Aw & Aw & Aw & - & Aw & Aw & Aw & - \\
\hline & Formoso & & Aw & - & Aw & Aw & Aw & - & Aw & Aw & $\mathrm{Aw}$ & - & Aw & Aw & Aw & - & Aw & Aw & Aw & - \\
\hline & João Pinheiro & & Aw & - & $\mathrm{Aw}$ & Aw & Aw & - & Aw & Aw & Aw & - & Aw & Aw & Aw & - & Aw & Aw & Aw & - \\
\hline & Paracatu & & Aw & - & $\mathrm{Aw}$ & Aw & Aw & - & Aw & Aw & Aw & - & Aw & Aw & Aw & - & Aw & Aw & Aw & - \\
\hline & Unaí & & Aw & - & Aw & Aw & Aw & - & Aw & Aw & Aw & - & Aw & Aw & Aw & - & Aw & Aw & Aw & - \\
\hline \multirow{3}{*}{ 多 } & Espinosa & \multirow{3}{*}{$0,5581 * *$} & BSh & - & BSh & BSh & BSh & - & BSh & BSh & BSh & - & BSh & BSh & BSh & - & BSh & BSh & BSh & - \\
\hline & Januária & & Aw & - & Aw & Aw & Aw & - & Aw & Aw & Aw & - & Aw & Aw & Aw & - & Aw & Aw & Aw & - \\
\hline & Moçambinho & & Aw & - & Aw & BWh & Aw & - & Aw & BWh & BWh & - & Aw & BWh & BWh & - & Aw & BWh & BWh & - \\
\hline
\end{tabular}




\section{ISSN: 2237-8642 (Eletrônica)}

\begin{tabular}{|c|c|c|c|c|c|c|c|c|c|c|c|c|c|c|c|c|c|c|c|c|}
\hline & Monte Azul & & BWh & - & BSh & BSh & BSh & - & BSh & BSh & BSh & - & BSh & BSh & BSh & - & BSh & BSh & BSh & - \\
\hline & Montes Claros & & Aw & - & Aw & Aw & Aw & - & Aw & Aw & Aw & - & Aw & Aw & Aw & - & Aw & Aw & Aw & - \\
\hline & Pirapora & & Aw & - & Aw & $A w$ & Aw & - & $A w$ & Aw & Aw & - & Aw & Aw & Aw & - & Aw & $A w$ & Aw & - \\
\hline & Salinas & & Aw & - & $A w$ & $A w$ & $A w$ & - & $A w$ & $A w$ & $A w$ & - & $A w$ & Aw & BWh & - & $A w$ & $A w$ & BWh & - \\
\hline \multirow{3}{*}{ 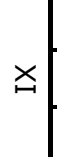 } & Carbonita & \multirow{3}{*}{ 0,0037* } & Aw & $\mathrm{Ba}$ & Aw & Aw & Aw & $\mathrm{Aa}$ & Aw & Aw & Aw & $\mathrm{Aa}$ & Aw & Aw & Aw & $\mathrm{Aa}$ & Aw & $\mathrm{Aw}$ & Aw & $\mathrm{Aa}$ \\
\hline & Itamarandiba & & Cwa & $\mathrm{Aa}$ & Aw & Aw & Aw & $A b$ & Aw & Aw & $A w$ & $A b$ & $A w$ & Aw & Aw & $A b$ & Aw & $A w$ & Aw & $A b$ \\
\hline & Pedra Azul & & Aw & $\mathrm{Ba}$ & Aw & Aw & Aw & $\mathrm{Aa}$ & $A w$ & Aw & $A w$ & $\mathrm{Aa}$ & $A w$ & BWh & BWh & $\mathrm{Bb}$ & Aw & $A w$ & BWh & $A a b$ \\
\hline
\end{tabular}

FATOR A = Estações meteorológicas; FATOR $D=$ Cenários $(R C P ' s) ; B L O C O=$ Projeções; ANOVA = probabilidade do teste $\mathrm{F}$ da $A N O V A ; * *=$ não significativo pelo teste $\mathrm{F} ; *=$ significativo pelo teste $\mathrm{F}$, ambos a $5 \%$ de probabilidade; $\mathrm{T}=$ Resultado do teste de Tukey; $-=$ nas classificações significa ausência de classificação por falta de dados; Médias seguidas pelas mesmas letras minúsculas na linha, para os RCP's, e maiúsculas na coluna, para as estações meteorológicas, não diferem entre si pelo teste Tukey $(a=0,05)$. 0 teste de Tukey foi aplicado somente nos casos em que o teste $\mathrm{F}$ foi significativo. 


\subsection{CLASSIFICAÇÃO CLIMÁTICA DE THORNTHWAITE}

Pela classificação climática de Thornthwaite, considerando o clima presente (1981-2010) (Figura 4), foi possível identificar 25 tipos climáticos em MG, diferente do número de tipos climáticos obtidos com o SCC de Köppen (Figura 2), o que é esperado em função da maior sensibilidade dos critérios de classificação do SCC de Thornthwaite (ROLIM et al., 2007).

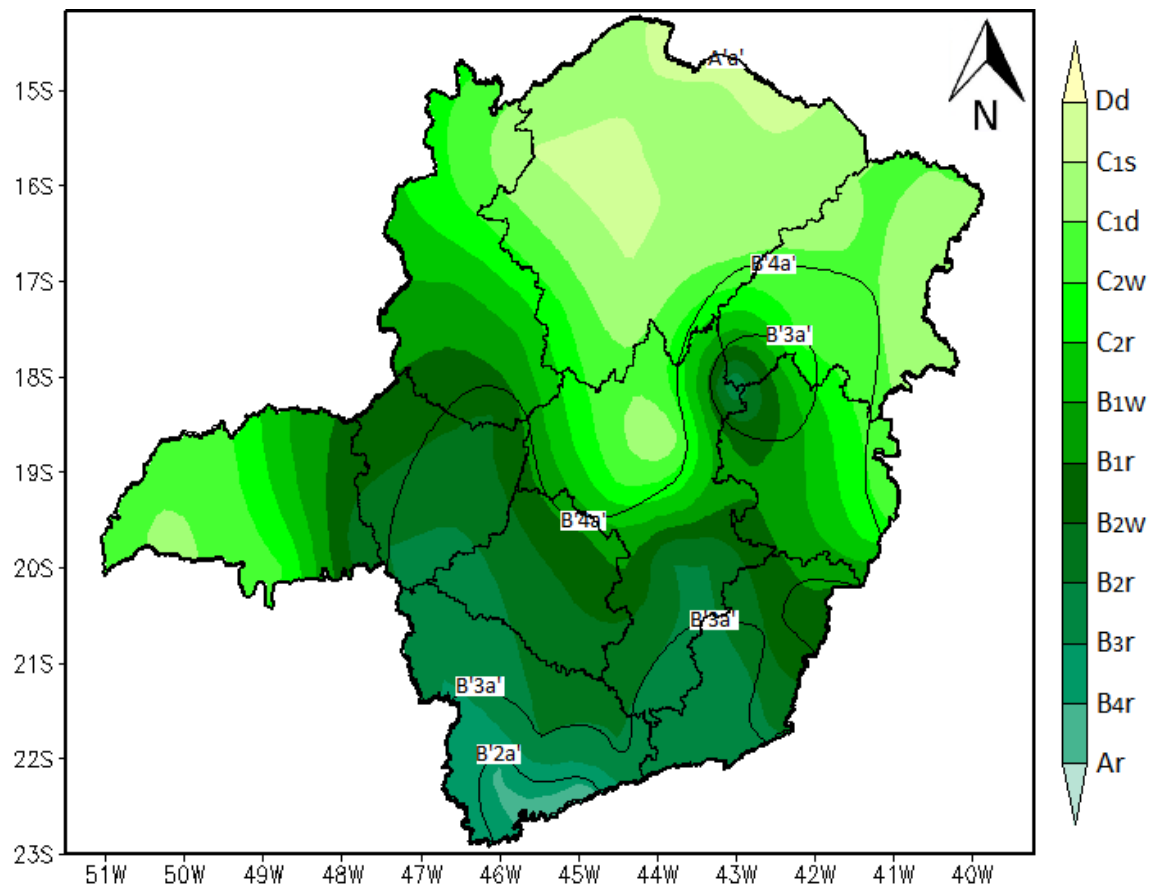

Figura 4 - Classificação climática de Thornthwaite para Minas Gerais considerando o clima presente (1981-2010). As linhas indicam os limites climáticos para as principais mudanças de temperatura e umidade, indicados pelos climas com fator hídrico $\mathrm{B}^{\prime} 2 \mathrm{a}$, $B^{\prime} 3 a^{\prime}, B^{\prime} 4 a^{\prime}$ e $A^{\prime} a^{\prime}$.

A comparação entre os SCC evidencia maior sensibilidade no método de Thornthwaite, corroborando com os resultados de Rolim et al. (2007) para São Paulo. Dessa forma, o SCC de Thornthwaite pode ser útil em estudos de mesoescala ou topoescala, onde os efeitos da topografia interferem diretamente nos elementos climáticos e, consequentemente, no planejamento das atividades agrícolas, ambientais e florestais regionais (ROLIM et al., 2007; ARAÚJO et al., 2012). Comparando-se os dois SCC (Tabela 6), em alguns casos verificou-se que não existe equivalência entre os tipos climáticos determinados por eles. Resultado similar foi obtido por Rolim et al. (2007) para o estado de São Paulo. Considerando o presente estudo, por exemplo, um tipo climático B1rB'4a' foi classificado, nas mesmas estações meteorológicas, como Cwa e Aw pelo SCC de Köppen. Essa não similaridade entre os dois SCC é esperada e ocorre em função das variáveis e da metodologia de classificação distintas em cada SCC. 
Tabela 6 - Comparação entre os sistemas de classificação climática de Köppen e de Thornthwaite (1948) para Minas Gerais considerando o clima presente (1981-2010).

\begin{tabular}{|c|c|c|c|c|c|c|}
\hline \multirow{2}{*}{$\frac{\text { Köppen }}{\text { Cwb }}$} & \multicolumn{6}{|c|}{ Thornthwaite } \\
\hline & $\operatorname{ArB}^{\prime} 2 a^{\prime}$ & B4rB'2a' & - & - & - & - \\
\hline Cwa & B1rB'4a' & B2rB'3a' & $\mathrm{B} 2 \mathrm{rB}^{\prime} 4 \mathrm{a}^{\prime}$ & B3rB'2a' & B3rB'3a' & C2wB'3a' \\
\hline \multirow{2}{*}{ Aw } & B1rB'4a' & B1wA'a' & $B 1 w B^{\prime} 4 a^{\prime}$ & B2rA'a' & $B 2 r B^{\prime} 4 a^{\prime}$ & B3rB'4a' \\
\hline & $C 1 d A^{\prime} a^{\prime}$ & C1sA'a' & $\mathrm{C} 2 \mathrm{rB}^{\prime} 4 \mathrm{a}^{\prime}$ & C2wA'a' & $C 2 w B^{\prime} 4 a^{\prime}$ & DdA'a' \\
\hline BSh & $\mathrm{DdA}^{\prime} \mathrm{a}^{\prime}$ & - & - & - & - & - \\
\hline BWh & DdA'a' & - & - & - & - & - \\
\hline
\end{tabular}

Na Figura 4, há predominância de tipos climáticos úmidos (15 tipos), seguidos pelo subúmidos ( 8 tipos) e um único tipo superúmido e outro semiárido. O único tipo superúmido (ArB'2a') ocorre apenas em Maria da Fé (Estação 34), enquanto os tipos úmidos (B1 a B4) se concentram na porção centro sul do estado, abrangendo as regiões Central, Mata, Sul, Alto Parnaíba e Centro-Oeste (Regiões I, II, III, IV e VI, respectivamente). A porção norte do estado, que engloba as regiões Noroeste, Norte e Vales do Jequitinhonha/Mucuri (Regiões VII, VIII e IX, respectivamente), apresentou climas predominantemente subúmidos ( 1 e $\mathrm{C} 2$ ) e semiárido (DdA'a'), os quais ocorreram em Aimorés, Espinosa, Mocambinho e Monte Alegre.

As regiões do Triângulo Mineiro e do Rio Doce, diferentemente da Central que possui latitude similar (18 $8^{\circ} \mathrm{S}$ a $\left.20^{\circ} \mathrm{S}\right)$, possuem dominância de climas subúmidos ( 1 e $\mathrm{C} 2$ ), que ocorrem devido à influência da continentalidade desta região (ÁVILA et al., 2014; GARCIA et al., 2018) e ocorrência de solos mais profundos e relevos mais planos (MELLO; VIOLA, 2013). Isso indica que, apesar dos grandes volumes de precipitação da região (em torno de $1400 \mathrm{~mm}$ ) (SANTOS et al., 2017), as condições de relevo fazem com que as perdas por ETP sejam maiores, devido ao maior aquecimento da superfície em relevo plano.

Em conformidade com o SCC de Köppen, a diferenciação de tipos climáticos ocorre, principalmente, devido à variação espacial e temporal da precipitação e temperatura do ar de MG (REBOITA et al., 2015; GARCIA et al., 2018). No Noroeste, Norte e Vales do Jequitinhonha/Mucuri há temperatura mais elevada e menor precipitação comparada às demais regiões de MG (SANTOS et al., 2017). Nesse sentido, o aumento da temperatura eleva a ETP, enquanto a redução da precipitação resulta em uma situação de deficiência hídrica (SHAO et al., 2009), alterando os extratos do BHC, influenciando os cálculos dos índices climáticos (Ih, Iu e Ia) e na ocorrência de climas subúmidos e semiáridos nesta porção de MG (Figura 4).

Com relação ao clima futuro (P1, P2 e P3), assim como o observado no SCC de Köppen (Figura 3), há tendência de climas mais quentes e secos ao longo do século XXI, intensificado na P3 e no RCP 8.5 (Figura 5). Há um aumento dos tipos climáticos C2WA'a (subúmido quente, com DEF moderada no verão), C2wB'4a (subúmido quente, com DEF moderada no inverno), C1dA'a' (subúmido seco e quente, com pequeno EXC), C1sA'a' (Subúmido seco e quente, com EXC moderado no verão) e DdA'a' (Semiárido quente). Isso ocorre devido às projeções de aumento crescente e gradual da temperatura do ar associado aos extremos de precipitação para MG (SANTOS et al., 2017). Estas 
projeções refletem diretamente em mudanças da ETP (LEMOS FILHO et al., 2010), com aumento da DEF, do Ia e redução do EXC, com maior intensidade no Noroeste, Norte e Vales do Jequitinhonha e Mucuri no final do século XXI no RCP 8.5, assim como nas demais regiões de Minas Gerais (CARDOSO; JUSTINO, 2014; SANTOS et al., 2017).

Pela ANOVA e comparação de médias para os tipos climáticos obtidos com o SCC de Thornthwaite (Tabela 7), verificou-se que apenas o Triângulo Mineiro, Alto Parnaíba, Centro-Oeste e Noroeste de Minas apresentam alteração dos tipos climáticos entre as estações meteorológicas (Fator A), RCP's (Fator D) ao longo das projeções, sendo intensificadas na P3. Como exemplo, no Triângulo e Alto Parnaíba (em Uberaba e Araxá), assim como no Centro-Oeste (Bambuí), haverá alteração do tipo climático B2rB'4a (úmido quente, com pequena DEF) para C2wA'a' (subúmido quente, com DEF moderada no inverno) ao longo das projeções.

As regiões Central, Mata, Sul, Norte de Minas, Jequitinhonha/Mucuri e Rio Doce apresentaram variância não significativa entre as classificações, RCP's e projeções. Isso significa que, de maneira geral, o padrão das mudanças nos tipos climáticos ocorre de duas maneiras distintas: ou são suaves ou praticamente desprezíveis ao longo das projeções, ou ocorrem abruptamente já na P1 e mantem-se ao longo das projeções. Como exemplo, no Norte de Minas, especialmente em Januária e Salinas (estações 24 e 43, respectivamente), haverá alteração do tipo climático para $\mathrm{DdA}^{\prime} \mathrm{a}^{\prime}$ (semiárido quente) já na $\mathrm{P} 1$ considerando o RCP 2.6, mantendo-se até o final do século. Outro exemplo é Maria da Fé (Sul) que no clima presente possui o tipo climático ArB'2a' (superúmido temperado sem DEF), sendo projetado passar para B4rB'2a' (úmido temperado sem ou com pequena DEF) já na $\mathrm{P} 1$, mantendo-se assim durante praticamente todo o século XXI para o RCP 2.6. 


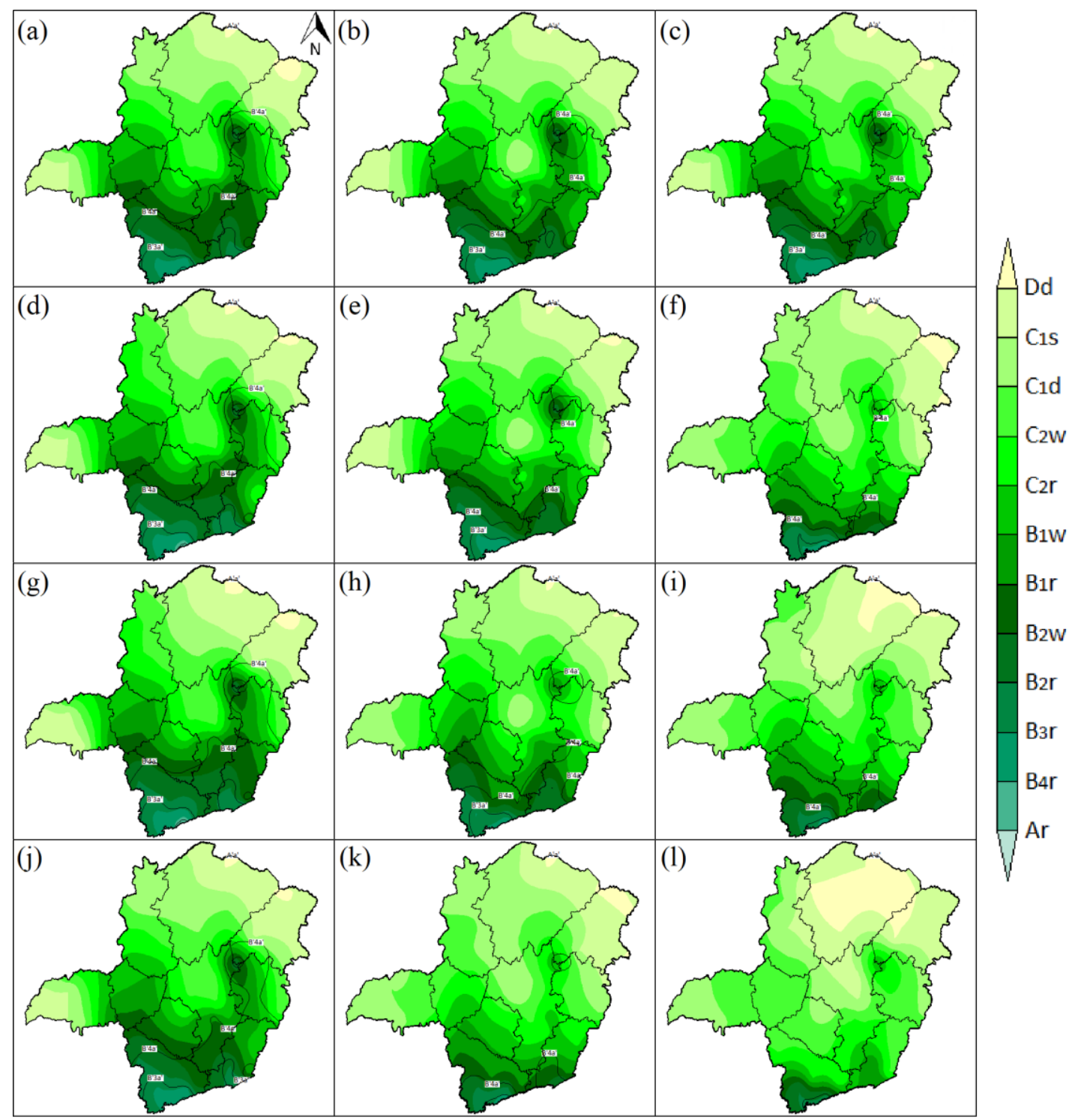

Figura 5 - Distribuição espacial da Classificação Climática de Thornthwaite para o estado de Minas Gerais a) RCP 2.6 para o período P1, b) RCP 2.6 para o período P2, c) RCP 2.6 para o período $\mathrm{P} 3$, d) RCP 4.5 para o período $\mathrm{P} 1$, e) RCP 4.5 para o período $\mathrm{P} 2$, f) $\mathrm{RCP}$ 4.5 para o período $\mathrm{P} 3, \mathrm{~g}) \mathrm{RCP} 6.0$ para o período $\mathrm{P} 1, \mathrm{~h}$ ) $\mathrm{RCP} 6.0$ para o período $\mathrm{P} 2, \mathrm{i}$ ) RCP 6.0 para o período $P 3, j$ ) RCP 8.5 para o período $P 1, k$ ) RCP 8.5 para o período $P 2$, I) RCP 8.5 para o período P3. 
Tabela 7 - Análise de variância (ANOVA) e comparação de médias pelo teste de Tukey para a classificação climática de Thornthwaite, considerando cada estação meteorológica, $\operatorname{RCP}^{\prime}$ (2.6, 4.5, 6.0 e 8.5) para o clima presente $(1981-2010)$ e projeções $(P 1=2011-2040$, 2041-2070, P3=2071-2100)

\begin{tabular}{|c|c|c|c|c|c|c|c|c|c|c|c|c|c|c|c|c|c|c|c|c|}
\hline \multicolumn{3}{|c|}{ FATOR A } & \multicolumn{18}{|c|}{ FATOR D } \\
\hline \multirow{3}{*}{ 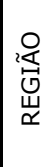 } & \multirow{3}{*}{ 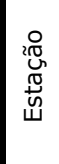 } & \multirow{3}{*}{ 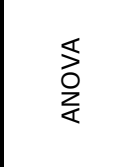 } & \multicolumn{2}{|c|}{ ATUAL } & \multicolumn{4}{|c|}{ RCP 2.6} & \multicolumn{4}{|c|}{ RCP 4.5} & \multicolumn{4}{|c|}{ RCP 6.0} & \multicolumn{4}{|c|}{ RCP 8.5} \\
\hline & & & \multirow{2}{*}{ BLOCO } & \multirow{2}{*}{ T } & \multicolumn{3}{|c|}{ BLOCO } & \multirow{2}{*}{$\mathrm{T}$} & \multicolumn{3}{|c|}{ BLOCO } & \multirow{2}{*}{$T$} & \multicolumn{3}{|c|}{ BLOCO } & \multirow{2}{*}{$\mathrm{T}$} & \multicolumn{3}{|c|}{ BLOCO } & \multirow{2}{*}{$\mathrm{T}$} \\
\hline & & & & & $\mathrm{P} 1$ & P2 & P3 & & $\mathrm{P} 1$ & P2 & P3 & & $\mathrm{P} 1$ & P2 & P3 & & $\mathrm{P} 1$ & P2 & P3 & \\
\hline \multirow{12}{*}{$\begin{array}{l}\times \\
0 \\
\sim\end{array}$} & 5 & \multirow{12}{*}{$0,9657 * *$} & в3rв'3а' & - & B2rB'3a' & B2rB'3a' & B2rB'3a' & - & B2rB'3a' & B2rB'4a' & B1rB'4a' & - & B2rB'3a' & B2rB'3a' & B1rB'4a' & - & B2rB'3a' & B1rB'4a' & B1rA'a' & - \\
\hline & 6 & & B2rB'4a' & - & B2wA'a' & B1wA'a' & B1wA'a' & - & B2wA'a' & B1wA'a' & B1wA'a' & - & B2wA'a' & B1wA'a' & B1wA'a' & - & B1wA'a' & B1wA'a' & |C2wA'a' & - \\
\hline & 8 & & B1rB'4a' & - & B1rA'a' & B1rA'a' & B1wA'a' & - & B1rA'a' & C2wA'a' & C2wA'a' & - & B1rA'a' & C2wA'a' & C2wA'a' & - & B1rA'a' & C2wA'a' & C2wA'a' & - \\
\hline & 14 & & C1sA'a' & - & C1dA'a' & C1dA'a' & C1dA'a' & - & C1dA'a' & C1dA'a' & C1dA'a' & - & C1dA'a' & C1dA'a' & C1dA'a' & - & C1dA'a' & C1dA'a' & D1dA'a' & - \\
\hline & 15 & & B3rB'2a' & - & B2rB'3a' & B2rB'3a' & B2rB'3a' & - & B2rB'3a' & B2rB'4a' & B1wB'4a' & - & B2rB'3a' & B1rB'4a' & B1wB'4a' & - & B2rB'3a' & B1wB'4a' & B1wA'a' & - \\
\hline & 18 & & B2rB'4a' & - & B1rB'4a' & B1rB'4a' & B1rA'a' & - & B1rB'4a' & B1wA'a' & C2wA'a' & - & B1rB'4a' & B1wA'a' & C2wA'a' & - & B1rB'4a' & C2wA'a' & |c2wA'a' & - \\
\hline & 21 & & B2rB'4a' & - & B1rB'4a' & B1rA'a' & B1rA'a' & - & B1rB'4a' & B1rA'a' & C2wA'a' & - & B1rB'4a' & B1rA'a' & C2wA'a' & - & B1rA'a' & C2wA'a' & C2wA'a' & - \\
\hline & 25 & & B1rB'4a' & - & B1rB'4a' & B1rB'4a' & B1wB'4a' & - & B1rB'4a' & B1wA'a' & C2wA'a' & - & B1rB'4a' & B1wA'a' & C2wA'a' & - & B1rB'4a' & C2wA'a' & |C2wA'a' & - \\
\hline & 42 & & C2wA'a' & - & C2wA'a' & C1sA'a' & C2wA'a' & - & C2wA'a' & C1sA'a' & C1sA'a' & - & |C2wA'a' & C1sA'a' & C1dA'a' & - & C2wA'a' & C1sA'a' & C1dA'a' & - \\
\hline & 46 & & B1wB'4a' & - & C2wA'a' & C2wA'a' & C2wA'a' & - & C2wA'a' & C2wA'a' & C2wA'a' & - & |C2wA'a' & C2wA'a' & C2wA'a' & - & C2wA'a' & C2wA'a' & C1sA'a' & - \\
\hline & 1 & & DdA'a' & - & DdA'a' & DdA'a' & - & - & DdA'a' & - & - & - & DdA'a' & - & - & - & |DdA'a' & - & - & - \\
\hline & 11 & & C2rB'4a' & - & C2wA'a' & C2wA'a' & C2wA'a' & - & C2wA'a' & C1sA'a' & C1sA'a' & - & |C2wA'a' & C1sA'a' & C1dA'a' & - & C2wA'a' & C1dA'a' & C1dA'a' & - \\
\hline \multirow{4}{*}{$\exists$} & 9 & \multirow{4}{*}{$0,6514 * *$} & B2rB'3a' & - & B1rB'4a' & B1rB'4a' & B1rB'4a' & - & B1rB'4a' & B1rB'4a' & B1rB'4a' & - & B1rB'3a' & B1rB'4a' & C2rB'4a' & - & B1rB'4a' & B1rB'4a' & C2wA'a' & - \\
\hline & 13 & & B2rB'4a' & - & B1rA'a' & B1rA'a' & B1rA'a' & - & B1rA'a' & B1rA'a' & B1rA'a' & - & B1rA'a' & B1rA'a' & B1rA'a' & - & B1rA'a' & B1rA'a' & |C2wA'a' & - \\
\hline & 27 & & Bзrв'3a' & - & B3rB'3a' & B2rB'3a' & B2rB'3a' & - & Bзrв'3a' & B2rB'4a' & B2rB'4a' & - & взrв'3a' & B2rB'4a' & B2rB'4a' & - & B3rB'3a' & B2rB'4a' & B1rA'a' & - \\
\hline & 49 & & B1rB'4a' & - & B1rB'4a' & C2rB'4a' & C2rB'4a' & - & C2wB'4a' & C2rA'a' & C2rA'a' & - & B1rB'4a' & C2rB'4a' & C2rA'a' & - & C2rB'4a' & C2rA'a' & C1dA'a' & - \\
\hline
\end{tabular}


Revista Brasileira de Climatologia

ISSN: 2237-8642 (Eletrônica)

\begin{tabular}{|c|c|c|c|c|c|c|c|c|c|c|c|c|c|c|c|c|c|c|c|c|}
\hline & 28 & \multirow{8}{*}{$0,4545^{* *}$} & B3rB'3a' & - & B3rB'3a' & B3rB'4a' & B3rB'4a' & - & B3rB'3a' & B2rB'4a' & B2rB'4a' & - & B3rB'3a' & B2rB'4a' & B2rB'4a' & - & B3rB'3a' & B2rB'4a' & B1rA'a' & - \\
\hline & 29 & & B2rB'4a' & - & B1rB'4a' & B1rB'4a' & B1rB'4a' & - & B2rB'4a' & B1rA'a' & B1rA'a' & - & B2rB'4a' & B1rA'a' & B1rA'a' & - & B1rB'4a' & B1rA'a' & C2wA'a' & - \\
\hline & 30 & & B2rB'4a' & - & B2rB'4a' & B2rB'4a' & B2rB'4a' & - & B2rB'4a' & B2rA'a' & B1rA'a' & - & B2rB'4a' & B1rB'4a' & B1rA'a' & - & B2rB'4a' & B1rA'a' & C2rA'a' & - \\
\hline & 31 & & ArB'2a' & - & B4rB'2a' & B4rB'2a' & B4rB'2a' & - & ArB'2a' & B4rB'2a' & B4rB'3a' & - & ArB'2a' & B4rB'2a' & B4rB'3a' & - & B4rB'2a' & B4rB'3a' & B3rB'4a' & - \\
\hline & 37 & & B3rB'3a' & - & B2rB'3a' & B2rB'4a' & B2rB'4a' & - & B2rB'3a' & B2rB'4a' & B2rB'4a' & - & B2rB'3a' & B2rB'4a' & B1rB'4a' & - & B2rB'4a' & B2rB'4a' & B1rA'a' & - \\
\hline & 41 & & B4rB'2a' & - & B3rB'3a' & B3rB'3a' & B3rB'3a' & - & B3rB'3a' & B3rB'3a' & B3rB'3a' & - & B3rв'3a' & B3rB'3a' & B2rB'4a' & - & Bзrв'3a' & B2rB'4a' & B2rA'a' & - \\
\hline & 44 & & B3rB'3a' & - & B2rB'4a' & B2rB'4a' & B2rB'4a' & - & B2rB'4a' & B2rB'4a' & B2rB'4a' & - & B2rB'4a' & B2rB'4a' & B1rA'a' & - & B2rB'4a' & B1rA'a' & B1rA'a' & - \\
\hline & 45 & & B3rB'4a' & - & B2rB'4a' & B2rA'a' & B2rA'a' & - & B2rB'4a' & B2rA'a' & B1rA'a' & - & B2rB'4a' & B2rA'a' & B1rA'a' & - & B2rA'a' & B2rA'a' & C2wA'a' & - \\
\hline \multirow{6}{*}{$\begin{array}{l}> \\
0 \\
\geq\end{array}$} & 10 & \multirow{6}{*}{$0,0001 *$} & C2wA'a' & $\mathrm{Ca}$ & C2wA'a' & C1sA'a' & C2wA'a' & \begin{tabular}{|c|c}
$\mathrm{Ca}$ \\
$\mathrm{b}$
\end{tabular} & C2wA'a' & C1sA'a' & C1sA'a' & $\mathrm{Cb}$ & C2wA'a' & C1sA'a' & C1sA'a' & $\mathrm{Cb}$ & C2wA'a' & C1sA'a' & C1dA'a' & $\mathrm{Cb}$ \\
\hline & 20 & & C2wA'a' & $\mathrm{Ca}$ & C1dA'a' & C1dA'a' & C1dA'a' & $\mathrm{Db}$ & C1dA'a' & C1dA'a' & C1dA'a' & $\mathrm{Cb}$ & C1sA'a' & C1dA'a' & C1dA'a' & $\mathrm{Cb}$ & C1dA'a' & C1dA'a' & C1dA'a' & $\mathrm{Cb}$ \\
\hline & 23 & & C2wA'a' & $\mathrm{Ca}$ & C1sA'a' & C1sA'a' & C1sA'a' & $\mathrm{Db}$ & C1sA'a' & C1sA'a' & C1dA'a' & $\mathrm{Cb}$ & C1sA'a' & C1dA'a' & C1dA'a' & $\mathrm{Cb}$ & C1sA'a' & C1dA'a' & C1dA'a' & $\mathrm{Cb}$ \\
\hline & 47 & & B2rA'a' & $\mathrm{Aa}$ & B1rA'a' & B1rA'a' & B1rA'a' & $A b$ & B1rA'a' & B1rA'a' & C2wA'a' & $A C$ & B1rA'a' & C2rA'a' & C2wA'a' & $B c d$ & B1rA'a' & C2wA'a' & C2wA'a' & $A B d$ \\
\hline & 2 & & B2rB'4a' & $\mathrm{Aa}$ & B1rA'a' & B1rA'a' & B1rA'a' & $A b$ & B1rA'a' & B1wA'a' & B1wA'a' & Acd & B1rA'a' & B1rA'a' & B1wA'a' & $A b c$ & B1rA'a' & B1wA'a' & C2wA'a' & $\mathrm{Ad}$ \\
\hline & 38 & & B2wB'4a' & $\mathrm{Ba}$ & B1wA'a' & B1wA'a' & B1wA'a' & $\mathrm{Bb}$ & B1wA'a' & B1wA'a' & C2wA'a' & $B b c$ & B1wA'a' & B1wA'a' & C2wA'a' & $\mathrm{Bbc}$ & B1wA'a' & C2wA'a' & C2wA'a' & $\mathrm{Bc}$ \\
\hline \multirow{4}{*}{5} & 4 & \multirow{4}{*}{$0,0038^{*}$} & B1rB'4a' & $\mathrm{Aa}$ & B1wA'a' & B1wA'a' & B1rA'a' & $\mathrm{Aa}$ & B1wA'a' & B1wA'a' & C2rA'a' & $\mathrm{ABa}$ & B1wA'a' & C2rA'a' & C2rA'a' & Aa & B1wA'a' & C2rA'a' & C2wA'a' & $\mathrm{Ba}$ \\
\hline & 7 & & B1rB'4a' & $\mathrm{Aa}$ & C2wA'a' & C2wA'a' & C2wA'a' & $\mathrm{Bb}$ & C2wA'a' & C2wA'a' & C2wA'a' & $\mathrm{Bb}$ & C2wA'a' & C2wA'a' & C2wA'a' & $A b$ & C2wA'a' & C2wA'a' & C2sA'a' & $\mathrm{Bb}$ \\
\hline & 16 & & B1rB'4a' & $\mathrm{Aa}$ & B1wA'a' & C2wA'a' & C2wA'a' & $\begin{array}{c}A B \\
a\end{array}$ & B1wA'a' & C2wA'a' & C2wA'a' & $A B a$ & B1wA'a' & C2wA'a' & C2wA'a' & Aa & B1wA'a' & C2wA'a' & C2wA'a' & $\mathrm{Ba}$ \\
\hline & 35 & & B2rB'4a' & $\mathrm{Aa}$ & B1rB'4a' & B1rA'a' & C2rA'a' & $A b$ & B1rB'4a' & B1rB'4a' & C2rA'a' & $A b$ & B1rB'4a' & C2rA'a' & C2wA'a' & $A b$ & B1rB'4a' & B2rB'4a' & - & $\mathrm{Aa}$ \\
\hline \multirow{5}{*}{$s$} & 3 & \multirow{5}{*}{ 0,0358* } & C1sA'a' & $\mathrm{Bb}$ & C1dA'a' & C1dA'a' & C1dA'a' & $\begin{array}{c}\mathrm{Ba} \\
\mathrm{b}\end{array}$ & C1dA'a' & C1dA'a' & C1dA'a' & Bab & B1rA'a' & C1dA'a' & C1dA'a' & $\mathrm{Aa}$ & C1dA'a' & C1dA'a' & DdA'a' & Aab \\
\hline & 19 & & C2wA'a' & $\begin{array}{c}A B \\
a\end{array}$ & C1sA'a' & C1sA'a' & C1sA'a' & $\mathrm{Bb}$ & C1sA'a' & C1dA'a' & C1dA'a' & Bab & C1sA'a' & C1dA'a' & C1dA'a' & Bab & C1sA'a' & C1dA'a' & C1dA'a' & Aab \\
\hline & 26 & & B1wA'a' & $\mathrm{Aa}$ & C2sA'a' & C2wA'a' & C2wA'a' & $\begin{array}{c}A a \\
b\end{array}$ & C2wA'a' & C2wA'a' & C1sA'a' & $A B b$ & C2wA'a' & C2wA'a' & C1sA'a' & $A B b$ & C2wA'a' & C1sA'a' & C1sA'a' & $A b$ \\
\hline & 36 & & B1wA'a' & $\mathrm{Aa}$ & C2wA'a' & C2wA'a' & C2wA'a' & \begin{tabular}{|c}
$A a$ \\
$b$ \\
\end{tabular} & C2wA'a' & C2wA'a' & C2wA'a' & Aab & C2wA'a' & C2wA'a' & C1sA'a' & $A B b$ & C2wA'a' & C2wA'a' & C1sA'a' & $A b$ \\
\hline & 48 & & C2wA'a' & $A B$ & C1sA'a' & C1sA'a' & C1sA'a' & $\mathrm{Bb}$ & C2wA'a' & C1sA'a' & C1sA'a' & $\mathrm{ABa}$ & C2wA'a' & C1sA'a' & C1dA'a' & $\mathrm{ABa}$ & C1sA'a' & C1sA'a' & C1dA'a' & $A b$ \\
\hline
\end{tabular}




\begin{tabular}{|c|c|c|c|c|c|c|c|c|c|c|c|c|c|c|c|c|c|c|c|c|}
\hline & & & & $a$ & & & & & & & & b & & & & b & & & & \\
\hline \multirow{7}{*}{ 实 } & 17 & \multirow{7}{*}{$0,6889 * *$} & DdA'a' & - & DdA'a' & - & - & - & - & - & - & - & - & - & - & - & - & - & - & - \\
\hline & 24 & & C1dA'a' & - & DdA'a' & DdA'a' & DdA'a' & - & DdA'a' & DdA'a' & DdA'a' & - & DdA'a' & DdA'a' & DdA'a' & - & DdA'a' & DdA'a' & DdA'a' & - \\
\hline & 32 & & DdA'a' & - & DdA'a' & DdA'a' & DdA'a' & - & DdA'a' & DdA'a' & DdA'a' & - & DdA'a' & DdA'a' & - & - & DdA'a' & DdA'a' & DdA'a' & - \\
\hline & 33 & & DdA'a' & - & DdA'a' & DdA'a' & - & - & DdA'a' & - & - & - & DdA'a' & - & - & - & DdA'a' & - & - & - \\
\hline & 34 & & C1sA'a' & - & C1dA'a' & C1dA'a' & C1dA'a' & - & C1dA'a' & C1dA'a' & C1dA'a' & - & C1sA'a' & C1dA'a' & DdA'a' & - & C1dA'a' & C1dA'a' & DdA'a' & - \\
\hline & 40 & & C1sA'a' & - & C1dA'a' & C1dA'a' & C1dA'a' & - & C1dA'a' & C1dA'a' & C1dA'a' & - & C1dA'a' & C1dA'a' & DdA'a' & - & C1dA'a' & C1dA'a' & DdA'a' & - \\
\hline & 43 & & C1dA'a' & - & DdA'a' & DdA'a' & DdA'a' & - & DdA'a' & DdA'a' & DdA'a' & - & DdA'a' & DdA'a' & - & - & DdA'a' & DdA'a' & - & - \\
\hline \multirow{3}{*}{$\underset{\bullet}{\bullet}$} & 12 & \multirow{3}{*}{$0,1722^{*}$} & C2 $2 \mathrm{wB}^{\prime} 4 \mathrm{a}^{\prime}$ & - & C1sA'a' & C1dA'a' & C1dA'a' & - & C1sA'a' & C1dA'a' & C1dA'a' & - & C1sA'a' & C1dA'a' & C1dA'a' & - & C1sA'a' & C1dA'a' & DdA'a' & - \\
\hline & 22 & & C2wB'3a' & - & C2wB'4a' & C2wB'4a' & C2wB'4a' & - & $C 2 w B^{\prime} 4 a^{\prime}$ & C2wB'4a' & C1sA'a' & - & $\begin{array}{c}\begin{array}{c}C 2 \mathrm{wB}^{\prime} 4 \\
a^{\prime}\end{array} \\
\end{array}$ & C2wB'4a' & C1dA'a' & - & $\begin{array}{c}\begin{array}{c}C 2 w^{\prime} 4 \\
a^{\prime}\end{array} \\
\end{array}$ & C1sA'a' & C1dA'a' & - \\
\hline & 39 & & C1dA'a' & - & DdA'a' & DdA'a' & DdA'a' & - & DdA'a' & DdA'a' & DdA'a' & - & DdA'a' & DdA'a' & DdA'a' & - & DdA'a' & DdA'a' & DdA'a' & - \\
\hline
\end{tabular}

FATOR A = Estações meteorológicas; FATOR D = Cenários (RCP's); BLOCO = Projeções; ANOVA = probabilidade do teste $\mathrm{F}$ da ANOVA; $* *$ $=$ não significativo pelo teste $\mathrm{F} ; *=$ significativo pelo teste $\mathrm{F}$, ambos a $5 \%$ de probabilidade; $\mathrm{T}=$ Resultado do teste de Tukey; $-=$ nas classificações, significa ausência de classificação por falta de dados; Médias seguidas pelas mesmas letras minúsculas na linha, para os RCP's, e maiúsculas na coluna, para as estações meteorológicas, não diferem entre si pelo teste Tukey $(a=0,05)$. 0 teste de Tukey foi aplicado somente nos casos em que o teste $\mathrm{F}$ foi significativo. 


\section{CONCLUSÃO}

Para o clima atual, Minas Gerais apresenta cinco tipos climáticos pela classificação climática de Köppen, sendo dois temperados quentes (Cwb, Cwa), um tropical (Aw) e dois semiáridos (BSh e BWh) e vinte e cinco por Thornthwaite, sendo considerado o SCC mais sensível em detectar os tipos climáticos.

Independente do SCC, Minas Gerais apresenta duas características climáticas bem definidas ao longo das projeções de clima futuro: as regiões centrais não apresentam alterações no SCC para P1 (2011 a 2040), mas apresentam para a P2 e P3 (2041-2100) e as regiões no norte e sul do estado, apresentam alterações para tipos climáticos predominantemente quentes e secos já na P1 (2011 a 2040).

As classificações climáticas são sensíveis à influência do relevo. No SCC de Köppen isso ocorre diretamente pelos valores da temperatura do ar, pois uma região com menor temperatura inserida em uma região de maior área, provavelmente tem essa característica devido à topografia. É interessante destacar que a altitude é um elemento de controle climático, pois devido ao efeito da gravidade, a densidade da atmosfera diminui com a altura. Assim, se está do nível médio do mar, menos gases são encontrados quanto mais distante em altura. Portanto, sobre uma montanha a atmosfera é mais rarefeita de forma que o aquecimento do ar é menor. No SCC de Thornthwaite, a influência do relevo ocorre na temperatura, que por sua vez influência a evapotranspiração, o extrato do $\mathrm{BHC}$ e os índices utilizados na classificação.

\section{AGRADECIMENTOS}

Ao INMET e CMIP5 pelos dados utilizados. À Fundação de Amparo à Pesquisa do Estado de Minas Gerais (FAPEMIG) pelo apoio financeiro. Ao Conselho Nacional de Desenvolvimento Científico e Tecnologico (CNPq) pelas bolsas concedidas.

\section{REFERÊNCIAS BIBLIOGRÁFICAS}

ALVARES, C. A.; STAPE, J. L.; SENTELHAS, P. C.; GOLÇALVES, J. L. M.; SPAROVEK, G. Köppen's climate classification map for Brasil. Meteorologische Zeitschrift, v. 22, n. 6, p. 711-728, 2014.

ARAÚJO, R. F. de; MATRICARDI, E. A. T.; NAPPO, M. E. Zoneamento ecológico de pequena escala para espécies florestais tradicionais no Distrito Federal. Floresta, v. 42, n. 2, p. 421-430, 2012.

ÁVILA, L. F.; MELLO, C. R. de; YANAGI, S. de N. M; NETO, O. B. S. Tendência de temperaturas mínimas e máximas do ar no estado de Minas Gerais. Pesquisa Agropecuária Brasileira, v. 49, n. 4, p. 247-256, 2014.

CARDOSO, G. M.; JUSTINO, F. Simulação dos componentes da evapotranspiração sob condições climáticas atuais e de cenários climáticos futuros de aquecimento global com o uso de modelos de clima-vegetação. Revista Brasileira de Meteorologia, v. 29, p. 85-95, 2014.

CAMARGO, A. P.; MARIN, F. R.; SENTELHAS, P. C. Ajuste da equação de Thornthwaite para estimar a evapotranspiração potencial em climas áridos e 
superúmidos, com base na amplitude térmica diária. Revista Brasileira de Agrometeorologia, v. 7, n. 2, p. 251-257, 1999.

CUNHA, A. R.; MARTINS, D. Classificação climática para os municípios de Botucatu e São Manuel, SP. Irriga, v. 14, n. 1, p. 1-11, 2009.

FABRES, T. M. Classificação climática segundo Köppen e Thornthwaite e caracterização edafoclimática referente à região de Santa Maria, RS. 2009. 127 f. Dissertação (Mestrado em Agronomia) - Escola Superior de Agricultura "Luiz de Quiroz", Universidade de São Paulo, Piracicaba, 2009.

FERNANDEZ, J. P. R.; FRANCHITO, S. H.; BRAHMANANDA RAO, V.; LLOPART, M. Changes in Koppen-Trewartha climate classification over South America from RegCM4 projections. Atmospheric Science Letters, v. 18, p. 427-434, 2017.

FERREIRA, D. F. Sisvar: A computer statistical analysis system. Ciência e Agrotecnologia, v.35, n.6, p.1039-1042, 2011.

GARCIA, S. R.; SANTOS, D. F.; MARTINS, F. B.; TORRES, R. R. Aspectos climatológicos associados ao cultivo da oliveira (Olea europaea L.) em Minas Gerais. Revista Brasileira de Climatologia, v. 22, p. 188-209, 2018.

GONÇALVES, J. L. de M.; ALVARES, C. A.; HIGA, A. R.; SILVA, L. D.; ALFENAS, A. C.; STAHL, J.; FERRAZ, S. F. de B.; LIMA, W. de P.; BRANCALION, P. H. S.; HUBNER, A; BOUILLET, J. P. D.; LACLAU, J. P.; NOUVELLON, Y., EPRON, D. Integrating genetic and silvicultural strategies to minimaze abiotic and biotic constraints in Brazilian eucalypt plantations. Forest Ecology and Managenment, v. 301, p. 6-27, 2013.

IBGE - Instituto Brasileiro de Geografia e Estatística. População de Minas Gerais. Brasília, 2016.2 Disponível em:<http://www.ibge.gov.br/estadosat/perfil.php?sigla=mg>. Acesso em: 10 de abril de 2017.

KÖPPEN, W. Das geographische System der Klimate. In: KÖPPEN, W.; GEIGER, R. (Eds): Handbuch der Klimatologie. Berlin: Gebrüder Bornträger, 1936. Banda 1, Parte C, p. 1-44.

KOTTEK, M.; GRIESER, J.; RUDOLF, B.; RUBEL, F. World map of the KöppenGeiger climate classification updated. Meteorologische Zeitschrift, v. 15, n. 3, p. 259-263, 2006.

LeMOS FILHO, L. C. A.; CARVAlho, L. G.; EVANGelistA, A. W. P.; ALVES JÚNIOR, J. Análise espacial da influência dos elementos meteorológicos sobre a evapotranspiração de referência em Minas Gerais. Revista Brasileira de Engenharia Agrícola e Ambiental, v. 1, p. 1294-1303, 2010.

MELLO, C. R. de; VIOLA, M. R. Mapeamento de chuvas intensas no Estado de Minas Gerais. Revista Brasileira de Ciência do Solo, v. 37, p. 37-44, 2013.

NÓBREGA, R. S. Um pensamento crítico sobre classificações climáticas: de Köppen até Strahler. Revista Brasileira de Geografia Física, v. 3, p. 18-22, 2010.

PEREIRA, A. R. Simplificando o balanço hídrico de Thornthwaite-Mather. Bragantia, v. 64, n. 2, p. 311-313, 2005.

REBOITA, M. S.; RODRIGUES, M.; SILVA, L. F.; ALVES, M. A. Aspectos climáticos de Minas Gerais. Revista Brasileira de Climatologia, v. 17, p. 206-226, 2015. 
REBOITA, M. S.; AMARO, T.R.; SOUZA, M.R.de. Winds: intensity and power density simulated by RegCM4 over South America in presente and future climate. Climate Dynamics, v. 51, n. 1-2, p. 187-205, 2017.

ROLIM, G. S.; CAMARGO, M. B. P.; LANIA, D. G.; MORAES, J. F. L. Classificação climática de Köppen e de Thornthwaite e sua aplicabilidade na determinação de zonas agroclimáticas para o estado de São Paulo. Bragantia, v. 66, n. 4, p. 711720, 2007.

SÁ JÚNIOR, A. de. Aplicação da classificação de Köppen para o zoneamento climático do estado de Minas Gerais. 2009. 101 f. Dissertação (Mestrado) Universidade Federal de Lavras, Lavras, 2009.

SANTOS, D. F. dos. Impacto das mudanças climáticas no zoneamento de aptidão climática das principais frutíferas de clima temperado nas regiões sul e sudeste do Brasil. 2018. 118 f. Dissertação (Mestrado em Meio Ambiente e Recursos Hídricos) - Universidade Federal de Itajubá, Itajubá, 2018.

SANTOS, L.F. dos; MARTINS, F.B.; GARCIA, S.R. Padrões climatológicos de precipitação e temperatura do ar associados ao rendimento do feijão comum em Minas Gerais. Revista Brasileira de Climatologia, v. XX, p.XX-XX, 2018 (no prelo).

SANTOS, D. F. dos; MARTINS, F. B.; TORRES, R. R. Impacts of climate projections on water balance and implications on olive crop in Minas Gerais. Revista Brasileira de Engenharia Agrícola e Ambiental, v. 21, n. 2, p. 77-82, 2017.

SENTELHAS, P. C.; DOS SANTOS, D. L.; MACHADO, R. E. Water deficit and water surplus maps for Brazil, based on FAO Penman-Monteith potential evapotranspiration. Revista Ambiente \& Água - Na Interdisciplinary Journal of Applied Science, v. 3, n. 3, p. 29-42, 2008.

SHAO, H.; CHU, L.; JALEEL, C. A.; MANIVANNAN, P.; PENNEERSELVAM, R.; SHAO, M. A. Understanding water deficit stress-induced changes in the basic metabolism of higher plants-biotechnologically and sustainably improving agriculture and the ecoenvironment in arid regions of the globe. Critical Reviews in Biotechnology, v. 29, n. 2, p. 131-151, 2009.

SILVA, P. H. M.; MIRANDA, A. C.; MORAES, M. L. T.; FURTADO, E. L.; STAPE, J. L.; ALVARES, C. A.; SENTELHAS, P. C.; MORI, E. S.; SEBBENN, A. M. Selecting for rust (Puccinia psidii) resistance in Eucalyptus grandis in São Paulo State, Brazil. Forest Ecology and Management, v. 303, p. 91-97, 2013.

STORCK, L.; GARCIA, D.C.; LOPES, S.J.; ESTEFANEL, V. Experimentação vegetal. Santa Maria: Ed. da UFSM, 2011. 200p.

TAYLOR, K. E.; STOUFFER, R. J.; MEEHL, G. A. Na overview of CMIP5 and the experimente desing. Bulletin of the American Meteorological Society, v. 93, p. 485-498, 2012.

THORNTHWAITE, C. W. An approach toward a rational classification of climate. Geography Review, v. 38, p. 55-94, 1948.

THORNTHWAITE, C. W.; MATHER, J. R. Instructions and tables for computing potential evapotranspiration and the water balance. Centerton, $\mathrm{Nj}$ : Drexel Institute of Technology - Laboratory of Climatology, 1957. 311p. Publications in Climatology, vol. X, n. 3. 
VAN VUUREN, D. P.; EDMONDS, J.; KAINUMA, M.; RIAHI, K.; THOMSON, A.; HIBBARD, K.; HURTT, G. C.; KRAM, T.; KREY, V.; LAMARQUE, J. F.; MAUSI, T.; MEINSHAUSEN, M.; NAKICENOVIC, N.; SMITH, S. J.; ROSE, S. K. The representative concentration pathways: an overview. Climatic Change, v. 109, p. 5-31, 2011.

VIANELLO, R. L.; ALVES, A. R. Meteorologia básica e aplicações. Viçosa, UFV: Imprensa Universitária, 2012. 449p.

WMO - World Meteorological Organization. Calculation of monthly and annual 30-year standard normals. Geneva, 1989. Technical document, n. 341; WCDP, n. 10 